\title{
The Genera of Fungi - G 4: Camarosporium and Dothiora
}

\author{
Pedro W. Crous ${ }^{1,2,3}$, and Johannes Z. Groenewald ${ }^{1}$
}

'Westerdijk Fungal Biodiversity Institute, Uppsalalaan 8, 3584 CT Utrecht, The Netherlands; corresponding author e-mail: p.crous@ westerdijkinstitute.nl

${ }^{2}$ Microbiology, Department of Biology, Utrecht University, Padualaan 8, $3584 \mathrm{CH}$ Utrecht, The Netherlands

${ }^{3}$ Department of Microbiology and Plant Pathology, Forestry and Agricultural Biotechnology Institute (FABI), University of Pretoria, P. Bag X20,

Pretoria 0028, South Africa

Abstract: The current paper represents the fourth contribution in the Genera of Fungi series, linking type species of fungal genera to their morphology and DNA sequence data. The present paper focuses on two genera of microfungi, Camarosporium and Dothiora, which are respectively epi- and neotypified. The genus Camarosporium is typified by $C$. quaternatum, which has a karstenula-like sexual morph, and phoma-like synasexual morph. Furthermore, Camarosporomyces, Foliophoma and Hazslinszkyomyces are introduced as new camarosporiumlike genera, while Querciphoma is introduced as a new phoma-like genus. Libertasomycetaceae is introduced as a new family to accommodate Libertasomyces and Neoplatysporoides. Dothiora, which is typified by $D$. pyrenophora, is shown to produce dothichiza- and hormonema-like synasexual morphs in culture, and $D$. cactacearum is introduced as a new species. In addition to their typification, ex-type cultures have been deposited in the Westerdijk Fungal Biodiversity Institute (CBS Culture Collection), and species-specific DNA barcodes in GenBank. Authors interested in contributing accounts of individual genera to larger multi-authored papers in this series should contact the associate editors listed on the List of Protected Generic Names for Fungi.

Key words:

DNA Barcodes

fungal systematics

ITS

LSU

typification

www.GeneraofFungi.org

Article info: Submitted: 15 April 2017; Accepted: 19 May 2017; Published: 23 May 2017.

\section{INTRODUCTION}

The present paper clarifies two generic names in the Genera of Fungi project (www.GeneraOfFungi.org; Crous et al. 2014a), which has the aim to revise the generic names of fungi accepted in Kirk et al. (2013). The two genera treated are supplemented with recently collected epi- or neotypes, which have been registered in MycoBank (Robert et al. 2013). Furthermore, in keeping with the one fungus = one name initiative for fungi (Hawksworth et al. 2011, Crous et al. 2015a), a single name is indicated for each genus.

The aim of the present contribution was to treat two problematic genera, namely Camarosporium, and Dothiora. In recent years, it has become clear that the Camarosporium morphology has evolved several times within Dothideomycetes, and that camarosporium-like morphs have phoma-like synasexual morphs, and pleospora-like sexual morphs. An additional complication lies in that some taxa only produce a single morph (e.g. see below in the present study), and thus it is almost impossible to clarify the generic identification without molecular data. Furthermore, the treatment of Phoma by Boerema et al. (2004) placed genetically distinct genera into one genus, while the same was true for the treatment of Pleospora by De Gruyter et al. (2013), and the assumption that the reference strains of Camarosporium included in Crous et al. (2006) were authentic for the name.
The genus Dothiora (based on D. pyrenophora) produces a Dothichiza asexual morph in culture, but the Dothideaceae has several genera that are dothiora-like in morphology, and that produce dothichiza-like asexual morphs in culture. Although we address the status of Dothiora in the present study, many additional collections are still required to resolve the generic boundaries of other old generic names in this family, particularly as we have observed hyaline, 1-septate ascospores to become muriformly septate with age, and eventually to even become pigmented. Given that these characters have traditionally been used to separate genera in the family (Sivanesan 1984, Thambugala et al. 2014), it now appears likely that there are far fewer genera in Dothideaceae than previously assumed (Crous \& Groenewald 2016).

\section{MATERIALS AND METHODS}

\section{Isolates}

Freshly collected twigs were placed in damp chambers, and incubated at room temperature (ca. $20^{\circ} \mathrm{C}$ ) for $1-2 \mathrm{~d}$. Single conidial or ascospore colonies were established from sporulating conidiomata or ascomata in Petri dishes containing $2 \%$ malt extract agar (MEA) as described earlier (Crous et al. 1991). After 1-2 d, single spores were picked up and transferred to fresh MEA plates. Colonies were sub-

\section{2017 International Mycological Association}

You are free to share - to copy, distribute and transmit the work, under the following conditions:

Attribution: $\quad$ You must attribute the work in the manner specified by the author or licensor (but not in any way that suggests that they endorse you or your use of the work).

Non-commercial: $\quad$ You may not use this work for commercial purposes.

No derivative works: You may not alter, transform, or build upon this work.

For any reuse or distribution, you must make clear to others the license terms of this work, which can be found at http://creativecommons.org/licenses/by-nc-nd/3.0/legalcode. Any of the above conditions can be waived if you get permission from the copyright holder. Nothing in this license impairs or restricts the author's moral rights. 
cultured onto $2 \%$ potato dextrose agar (PDA), oatmeal agar (OA), MEA (Crous et al. 2009), autoclaved pine needles on $2 \%$ tap water agar (PNA) (Smith et al. 1996), and incubated at $25{ }^{\circ} \mathrm{C}$ under continuous near-ultraviolet light to promote sporulation. Reference strains and specimens are maintained at the Westerdijk Fungal Biodiversity Institute (CBS Culture Collection) in Utrecht, The Netherlands.

\section{DNA isolation, amplification and analyses}

Genomic DNA was extracted from fungal colonies growing on MEA using the Wizard $®$ Genomic DNA purification kit (Promega, Madison, WI), according to the manufacturer's protocol. The primers V9G (de Hoog \& Gerrits van den Ende 1998) or ITS5 (White et al. 1990) and LR5 (Vilgalys \& Hester 1990) were used to amplify for all isolates part of the nuclear rDNA operon (ITS) spanning the 3' end of the 18S nrRNA gene, the first internal transcribed spacer (ITS1), the $5.8 \mathrm{~S}$ nrRNA gene, the second ITS region (ITS2) and approximately $900 \mathrm{bp}$ of the 5 ' end of the $28 \mathrm{~S}$ nrRNA gene. The primers ITS4 (White et al. 1990) and LROR (Vilgalys \& Hester 1990) were used as internal sequence primers to ensure good quality sequences over the entire length of the amplicon. Part of the beta-tubulin gene (tub2) was amplified and sequenced for selected isolates using T1 (O'Donnell \& Cigelnik 1997) or Bt-2a and Bt-2b (Glass \& Donaldson 1995). In addition, part of the first or second half of the translation elongation factor 1-alpha gene (tef1) was amplified and sequenced for selected isolates using EF1-728F (Carbone \& Kohn 1999) and EF2 (O'Donnell et al. 1998), and EF1-983F and EF12218R (Rehner \& Buckley 2005), respectively. Part of the $18 \mathrm{~S}$ small subunit nrRNA gene (SSU) was amplified and sequenced for selected isolates using NS1 and NS4 (White et al. 1990). Amplification conditions followed Cheewangkoon et al. (2008) and Quaedvlieg et al. (2012). The programme SeqMan Pro v. 13.0.0 (DNASTAR, Madison, WI) was used to obtain consensus sequences of each isolate. Blast searches using ITS and LSU sequences were performed for each isolate and the closest matches were retrieved from GenBank and included in the phylogenetic analyses. The SSU, tef1 and tub2 sequences were not included in phylogenetic analyses but were used in blast searches to confirm the species identification where possible. Multiple sequence alignments for ITS and LSU were generated using the online version of MAFFT (http://mafft.cbrc.jp/alignment/software/) and subsequent phylogenetic analyses were conducted using parsimony in PAUP v. $4.0 b 10$ (Swofford 2003) as described by Cheewangkoon et al. (2008). Sequence data were deposited in GenBank (Table 1) and the alignments and trees in TreeBASE (http://www.treebase.org).

\section{Morphology}

Slide preparations were mounted in clear lactic acid or water from colonies sporulating on the media previously mentioned. Sections of sporocarps were made by hand for examination purposes. Observations were made with a Nikon SMZ25 stereo-microscope, and with a Zeiss Axio Imager 2 light microscope using differential interference contrast (DIC) illumination and a Nikon DS-Ri2 camera and software. Colony characters and pigment production were noted after $2 \mathrm{wk}$ of growth on MEA, PDA and OA (Crous et al. 2009) incubated at $25^{\circ} \mathrm{C}$. Colony colours (surface and reverse) were rated according to the colour charts of Rayner (1970). Taxonomic novelties and new typifications were deposited in MycoBank (www.MycoBank.org; Crous et al. 2004).

\section{RESULTS}

\section{Phylogeny}

Four analyses were performed in this study; the first was based on a partial alignment of LSU (Fig. 1) to provide an overview phylogeny of the species and genera treated in the present study, whereas the remaining three alignments were ITS alignments representing Camarosporium and allied genera (Fig. 2), Dothidea and Dothiora (Fig. 3), and Paracamarosporium and Pseudocamarosporium (Fig. 4), respectively. The statistical parameters for these four phylogenies are summarised in Table 2.

The overview LSU phylogeny (Fig. 1) shows that Dothiora pyrenophorais nestled inside the Dothioraclade. Based on this LSU phylogeny, the Dothidea clade has a bootstrap support value of $99 \%$, but the Dothiora clade did not receive any bootstrap support. Libertasomyces and Neoplatysporoides formed a lineage distinct from other families included in the phylogeny and therefore a family name is introduced below to accommodate these genera. Camarosporium quaternatum clustered with Ochrocladosporium, but without any bootstrap support. Megablast searches of the NCBI GenBank nucleotide database failed to reveal any closer matches. Strains previously published as Camarosporium quaternatum (CBS 134.97 and CBS 483.95) proved to be neither conspecific nor congeneric with $C$. quaternatum and therefore new combinations are proposed to accommodate these two strains in Libertasomyces and the new genus Hazslinszkyomyces. Two species of Pleospora, P. flavigena and $P$. fallens, were not congeneric with Pleospora (based on $P$. herbarum) and therefore the new genera Camarosporomyces and Foliophoma are introduced to accommodate these taxa. The genus Hazslinszkyomyces is erected below to accommodate "Camarosporium" aloes, while Querciphoma is erected to accommodate "Coniothyrium" carteri. Several species treated until now as belonging to Camarosporium represent yet another new genus and family, which will be treated in elsewhere.

The ITS phylogeny of Camarasporium and allied genera (Fig. 2) resolved most of the included species, except for those in the "Camarosporium" clade where the ITS sequences were highly similar ( $97 \%$ similar and higher when compared to "Camarosporium" sp. 1 strain CPC 12441; a maximum of 14 nucleotides differences). Neoplatysporoides aloicola (GenBank KR476719) clustered inside Libertasomyces, compared to the LSU phylogeny (Fig. 1) where it was basal to the clade. The sister placement of $N$. aloicola (conidia brown, 1-septate) to $L$. myopori (conidia hyaline, aseptate) did not receive any bootstrap support and this species is therefore not combined into Libertasomyces. In the LSU phylogeny (Fig. 1), Camarosporomyces flavigenus clustered as a basal lineage in the Hazslinszkyomyces clade, and based on this position was first considered to be a species of that genus. However, the ITS sequence was clearly not congeneric with 
Hazslinszkyomyces (Fig. 2) and a new generic name is therefore introduced to accommodate it. Both the ITS and LSU sequences of this strain were confirmed by two independent DNA isolations, and subsequent PCR and sequencing.

The ITS phylogeny of Dothidea and Dothiora (Fig. 3) resolved the included species, although the lineages in Dothiora were poorly supported. Crous \& Groenewald (2016) used a combination of ITS, tef1 and tub2 to resolve the species in this genus. Dothiora cactacearum is introduced below as a new species related to $D$. buxi while the generic type species, $D$. pyrenophora, is shown to be sister to $D$. sorbi. In the Dothidea clade, three isolates representing $D$. ribesia were not conspecific with two isolates published by Thambugala et al. (2014) as Plowrightia ribesia and which are indicated in our phylogeny as "Dothidea sp." pending further elucidation.

The ITS phylogeny of Paracamarosporium and Pseudocamarosporium (Fig. 4) resolved most of the included Paracamarosporium species, but did not resolve species in the Pseudocamarosporium clade where the ITS sequences were highly similar (99\% similar and higher when compared to Pseudocamarosporium sp. 1 strain CPC 25926; a maximum of five nucleotides differences). "Camarosporium" mamanes (GenBankDQ885900) clustered as sister to Paracamarosporium psoraleae and therefore a new combination is provided for it in that genus.

\section{THE GENERA}

\section{Camarosporium complex}

Coniothyriaceae W.B. Cooke, Revista de Biol. 12: 289 (1983) [“1980-1983”].

Type species: Coniothyrium palmarum Corda 1840.

Genera included: Camarosporium, Camarosporomyces, Coniothyrium, Dimorphosporicola, Foliophoma, Hazslinszkyomyces, Neocamarosporium, Ochrocladosporium, Pseudoleptosphaeria.

Note: Camarosporiaceae Locq. 1984 is not validly published (Art. 39.1) in lacking a Latin diagnosis. However, the genus Camarosporium s. str. on which this family was based, falls in Coniothyriaceae (see De Gruyter et al. 2013) in our analysis, thus we refrain from validating Camarosporiaceae to accommodate Camarosporium.

Camarosporium Schulzer, Verh. K.K. Zool.-Bot. Ges. Wien 17: 717 (1867).

Classification: Coniothyriaceae, Pleosporales, Dothideomycetes.

Current generic circumscription: Ascomata pseudothecial, single or in clusters, subcorticolous, +/- globose, black, ostiolum central, short papillate and terete, without setae; ascomatal wall of textura angularis. Pseudoparaphyses numerous, filiform, cellular, multi-celled, branched, anasto- mosing, hyaline, smooth. Asci 8-spored, cylindrical, apically rounded, pedicel short and furcate, thick-walled, bitunicate, fissitunicate, inamyloid. Ascospores 6-celled, ellipsoidal, straight, muriform, golden, with 1-3 longitudinal septa, eguttulate, without a gelatinous sheath and appendages. Conidiomata dimorphic, pycnidial, subcorticolous, single to gregarious, partly caespitose, globose, ostiole central, terete, short papillate; conidiomatal wall few-layered, consisting of a textura globulosa-angularis with red brown, thick-walled, and smooth cells. Paraphyses and conidiophores absent. Conidiogenous cells formed from the inner cells of the pycnidial wall, doliiform, hyaline, thin-walled, annellidic. Conidia multicelled, muriformly septate, with one longitudinal or diagonal septum per cell and 1-2 per conidium, ellipsoidal, pyroid, clavate, straight to slightly curved, yellowish not brown, basal cell often paler or hyaline, wall golden. Synasexual morph: conidiomata separate, pycnidial, immersed to superficial on PNA, brown, globose, with 1-2 papillate ostioles, exuding a crystalline conidial mass. Conidiophores reduced to conidiogenous cells. Conidiogenous cells lining the inner cavity, hyaline, smooth, ampulliform. Conidia solitary, hyaline, smooth, subcylindrical, straight, rarely curved, apex obtuse, base truncate.

Type species: Camarosporium quaternatum (Hazsl.) Schulzer 1867.

Camarosporium quaternatum (Hazsl.) Schulzer, Verh. K.K. Zool.-Bot. Ges. Wien 17: 717 (1867).

(Fig. 5)

Basionym: Clinterium quaternatum Hazsl., Verh. K.K. Zool.-Bot. Ges. Wien 15: 450 (1865); as "Clinterium (quaternatum)"; type: Hazslinszky (1865: figs 9-12 - lectotype designated here, MBT376246; Hungary: near Budapest, private garden, on twigs of Lycium barbarum (Solanaceae), attached, corticated, 2 May 2016, L. Bartalos, det. R.K. Schumacher (CBS H-23064 - epitype designated here, MBT376247; CPC 31081 = CBS 142616 - culture exepitype, CPC 31518).

Synonyms: Cucurbitaria varians Hazsl., Verh. K.K. Zool.-Bot. Ges. Wien 15: 451 (1865); type: Hazslinszky (1865: figs 13-24 - lectotype designated here, MBT376243).

Karstenula varians (Hazsl.) Sacc., Syll. Fung. 2: 241 (1883).

Pleomassaria varians (Hazsl.) G. Winter, Rabenh. Krypt.-Fl., $2^{\text {nd }}$ edn 1(2): 552 (1886).

Camarosporium hendersonia Schulzer, Verh. K.K. Zool.-Bot. Ges. Wien 17: 716 (1867), nom. illegit. (Art. 53.1, based on C. quaternatum (Hazsl.) Schulzer 1867).

Camarosporium hazslinszkyi Sacc., Syll. Fung. 3: 468 (1884), nom. illegit. (Art. 53.1, based on C. quaternatum (Hazsl.) Schulzer 1867).

Additional material examined: Germany: Berlin, mixed forest, about $50 \mathrm{~m}$ asl, sand, acid, fresh, mesotroph, on twig of Daphne mezereum (Thymelaeaceae), 18 May 2013, R. Jarling, det. R.K. Schumacher (CBS H-23063, culture CPC $23216=$ CBS 142617).

Description: Ascomata pseudothecial, single or in clusters, subcorticolous, +/- globose with a flattened base, black, smooth, soft, thick, ostiole central, short papillate and terete, 
Table 1. Details of the strains included in the taxomic treatments or for which novel sequences were deposited in GenBank.

\begin{tabular}{|c|c|c|c|c|c|c|c|c|c|c|}
\hline \multirow[t]{2}{*}{ Species name } & \multirow{2}{*}{$\begin{array}{l}\text { Strain accession } \\
\text { number }{ }^{1,2}\end{array}$} & \multirow{2}{*}{$\begin{array}{l}\text { Collector and } \\
\text { collection date }\end{array}$} & \multirow[t]{2}{*}{ Host or substrate } & \multirow[t]{2}{*}{ Country } & \multicolumn{6}{|c|}{ GenBank accession number ${ }^{3}$} \\
\hline & & & & & ITS & LSU & $\begin{array}{l}\text { tef1 (first } \\
\text { part) }\end{array}$ & $\begin{array}{l}\text { tef1 (second } \\
\text { part) }\end{array}$ & tub2 & ssu \\
\hline $\begin{array}{l}\text { "Camarosporium" } \\
\text { arezzoensis }\end{array}$ & CPC 31420 & $\begin{array}{l}\text { R.K. Schumacher, } \\
22 \text { Jul. } 2016\end{array}$ & $\begin{array}{l}\text { Cytisus borysthenicus, } \\
\text { branch }\end{array}$ & Ukraine & KY929127 & KY929163 & - & - & - & - \\
\hline "Camarosporium" sp. 1 & CPC 12441 & W. Gams, Aug. 2005 & Sophora chrysophylla & USA: Hawaii & KY929128 & DQ377885 & - & - & - & - \\
\hline \multirow[t]{2}{*}{ “Camarosporium” sp. 2} & CPC 25960 & $\begin{array}{l}\text { R.K. Schumacher, } \\
21 \text { Dec. } 2014\end{array}$ & Caragana sp., twig & Finland & KY929129 & KY929164 & - & KY929198 & - & - \\
\hline & CPC 25962 & $\begin{array}{l}\text { R.K. Schumacher, } 1 \\
\text { Dec. } 2014\end{array}$ & Caragana sp., twig & Finland & KY929130 & KY929165 & - & KY929199 & - & - \\
\hline "Camarosporium" sp. 3 & CPC 31031 & $\begin{array}{l}\text { R.K. Schumacher, } \\
10 \text { May } 2016\end{array}$ & $\begin{array}{l}\text { Elaeagnus rhamnoides, } \\
\text { twig }\end{array}$ & Germany & KY929131 & KY929166 & - & - & - & - \\
\hline "Camarosporium" sp. 4 & CPC 31632 & $\begin{array}{l}\text { R.K. Schumacher, } \\
12 \text { Oct. } 2016\end{array}$ & Ulmus laevis, twig & Ukraine & KY929132 & KY929167 & - & - & - & - \\
\hline \multirow[t]{2}{*}{ "Camarosporium" sp. 5} & CPC 27667 & $\begin{array}{l}\text { A. Usichenko, } 25 \\
\text { May } 2015\end{array}$ & $\begin{array}{l}\text { Robinia pseudoacacia, } \\
\text { dead branch }\end{array}$ & Ukraine & KY929133 & KY929168 & - & - & - & - \\
\hline & СРС 30379 & $\begin{array}{l}\text { R.K. Schumacher, } 2 \\
\text { Apr. } 2016\end{array}$ & $\begin{array}{l}\text { Philadelphus } \\
\text { coronarius, twig }\end{array}$ & Germany & KY929134 & KY929169 & - & - & - & - \\
\hline \multirow[t]{3}{*}{$\begin{array}{l}\text { Camarosporium } \\
\text { quaternatum }\end{array}$} & CPC 23216 & $\begin{array}{l}\text { R.K. Schumacher, } \\
18 \text { May } 2013\end{array}$ & $\begin{array}{l}\text { Daphne mezereum, } \\
\text { twig }\end{array}$ & Germany & KY929135 & KY929170 & - & KY929200 & - & KY929122 \\
\hline & $\begin{array}{l}\text { CPC } 31081 \text { ET of Clinterium } \\
\text { quatermatum }\end{array}$ & $\begin{array}{l}\text { R.K. Schumacher, } 2 \\
\text { May } 2016\end{array}$ & Lycium barbarum, twig & Hungary & KY929136 & KY929171 & - & KY929201 & - & KY929123 \\
\hline & CPC 31518 & $\begin{array}{l}\text { R.K. Schumacher, } 2 \\
\text { May } 2016\end{array}$ & Lycium barbarum, twig & Hungary & KY929137 & KY929172 & - & KY929202 & - & KY929124 \\
\hline $\begin{array}{l}\text { Camarosporomyces } \\
\text { flavigenus }\end{array}$ & CBS $314.80^{\top}$ & K. Fodor, 1980 & Water & Romania & KY929138 & GU238076 & - & - & - & GU238217 \\
\hline Dothidea puccinioides & CBS 193.58 & - & Viburnum lantana & Switzerland & KY929139 & AY004342 & - & - & - & - \\
\hline \multirow[t]{3}{*}{ Dothidea ribesia } & CPC 30638 & R.K. Schumacher & Ribes uva-crispa, stem & Germany & KY929140 & KY929173 & KY929192 & - & KY929205 & - \\
\hline & CPC 30689 & $\begin{array}{l}\text { R.K. Schumacher, } 4 \\
\text { May } 2016\end{array}$ & Ribes rubrum, twig & Germany & KY929141 & KY929174 & KY929193 & - & KY929206 & - \\
\hline & CPC 30713 & $\begin{array}{l}\text { R.K. Schumacher, } 4 \\
\text { May } 2016\end{array}$ & Ribes rubrum, twig & Germany & KY929142 & KY929175 & KY929194 & - & KY929207 & - \\
\hline \multirow[t]{2}{*}{ Dothiora cactacearum } & $\begin{array}{l}\text { CBS } 142492=\text { CPC } \\
15585\end{array}$ & P.W. Crous, 2013 & Cactaceae (ornamental) & USA & KY929143 & KY929176 & KY929195 & - & KY929208 & - \\
\hline & CPC 15587 & P.W. Crous, 2013 & Cactaceae (ornamental) & USA & KY929144 & KY929177 & KY929196 & - & KY929209 & - \\
\hline \multirow[t]{2}{*}{ Dothiora pyrenophora } & CPC $30632^{\mathrm{NT}}$ & $\begin{array}{l}\text { R.K. Schumacher, } \\
27 \text { Apr. } 2016\end{array}$ & Sorbus aucuparia, twig & Germany & KY929145 & KY929178 & - & KY929203 & KY929210 & KY929125 \\
\hline & CPC 30634 & $\begin{array}{l}\text { R.K. Schumacher, } \\
27 \text { Apr. } 2016\end{array}$ & Sorbus aucuparia, twig & Germany & KY929146 & KY929179 & - & KY929204 & KY929211 & KY929126 \\
\hline Foliophoma fallens & CBS 161.78 & G.F. Laundon, 1978 & $\begin{array}{l}\text { Olea europaea, leaf } \\
\text { spot }\end{array}$ & $\begin{array}{l}\text { New } \\
\text { Zealand }\end{array}$ & KY929147 & GU238074 & - & - & - & GU238215 \\
\hline
\end{tabular}


GenBank accession number ${ }^{3}$

\begin{tabular}{|c|c|c|c|c|c|c|c|c|c|c|}
\hline \multirow[b]{2}{*}{ Species name } & \multirow[b]{2}{*}{$\begin{array}{l}\text { Strain accession } \\
\text { number } 1,2\end{array}$} & \multirow[b]{2}{*}{$\begin{array}{l}\text { Collector and } \\
\text { collection date }\end{array}$} & \multirow[b]{2}{*}{ Host or substrate } & \multirow[b]{2}{*}{ Country } & \\
\hline & & & & & ITS & LSU & $\begin{array}{l}\text { tef1 (first } \\
\text { part) }\end{array}$ & $\begin{array}{l}\text { tef1 (second } \\
\text { part) }\end{array}$ & tub2 & SSU \\
\hline & CBS 284.70 & $\begin{array}{l}\text { H.A. van der Aa, } \\
\text { Apr. } 1970\end{array}$ & $\begin{array}{l}\text { Nerium oleander, leaf } \\
\text { spot }\end{array}$ & Italy & KY929148 & GU238078 & - & 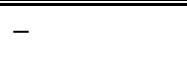 & - & GU238218 \\
\hline Hazslinszkyomyces aloes & $\begin{array}{l}\text { CBS } 136437=\text { CPC } \\
21572^{\top}\end{array}$ & $\begin{array}{l}\text { M.J. Wingfield, Sept. } \\
2012\end{array}$ & $\begin{array}{l}\text { Aloe dichotoma, dead } \\
\text { bark }\end{array}$ & South Africa & KF777142 & KF777198 & - & - & - & - \\
\hline Hazslinszkyomyces aptrootii & CBS $483.95^{\top}$ & $\begin{array}{l}\text { A. Aptroot, } 17 \text { Mar. } \\
1995\end{array}$ & Lycium sp. & Netherlands & KY929149 & DQ377884 & - & GU349044 & - & GU296141 \\
\hline \multirow[t]{2}{*}{ Hazslinszkyomyces lycii } & CPC $30998^{\top}$ & $\begin{array}{l}\text { R.K. Schumacher, } 2 \\
\text { May } 2016\end{array}$ & Lycium barbarum, twig & Hungary & KY929150 & KY929180 & - & - & - & - \\
\hline & CPC 31014 & $\begin{array}{l}\text { R.K. Schumacher, } 1 \\
\text { May } 2016\end{array}$ & Lycium barbarum, twig & Hungary & KY929151 & KY929181 & - & - & - & - \\
\hline Libertasomyces quercus & $\begin{array}{l}\text { CBS } 134.97=\text { INIFAT } \\
\text { C96/108 }\end{array}$ & $\begin{array}{l}\text { R.F. Castañeda, } 20 \\
\text { Jul. } 1996\end{array}$ & Quercus ilex, leaf litter & Spain & KY929152 & DQ377883 & KY929197 & - & KY929212 & - \\
\hline $\begin{array}{l}\text { Neocamarosporium } \\
\text { chersinae }\end{array}$ & CPC $27298^{\top}$ & $\begin{array}{l}\text { P.W. Crous, } 25 \text { May } \\
2015\end{array}$ & $\begin{array}{l}\text { Dead angulate tortoise } \\
\text { shell }\end{array}$ & South Africa & KY929153 & KY929182 & - & - & - & - \\
\hline Paracamarosporium fagi & CPC 31037 & $\begin{array}{l}\text { R.K. Schumacher, } \\
10 \text { May } 2016\end{array}$ & $\begin{array}{l}\text { Elaeagnus rhamnoides, } \\
\text { twig }\end{array}$ & Germany & KY929154 & KY929183 & - & - & - & - \\
\hline Paracamarosporium sp. 1 & CPC 30988 & $\begin{array}{l}\text { R.K. Schumacher, } 1 \\
\text { May } 2016\end{array}$ & Tilia platyphyllos, twig & Germany & KY929155 & KY929184 & - & - & - & - \\
\hline Pseudocamarosporium sp. 1 & CPC 25926 & $\begin{array}{l}\text { M.J. Wingfield, Nov. } \\
2014\end{array}$ & Erica sp. & South Africa & KY929156 & KY929185 & - & - & - & - \\
\hline \multirow[t]{6}{*}{ Pseudocamarosporium sp. 2} & CPC 25002 & R.K. Schumacher & Platanus sp., branch & Switzerland & KY929157 & KY929186 & - & - & - & - \\
\hline & CPC 25004 & R.K. Schumacher & Platanus sp., branch & Switzerland & KY929158 & KY929187 & - & - & - & - \\
\hline & CPC 25843 & $\begin{array}{l}\text { R.K. Schumacher, } \\
17 \text { Dec. } 2014\end{array}$ & Betula pendula, twig & Germany & KY929159 & KY929188 & - & - & - & - \\
\hline & CPC 27400 & $\begin{array}{l}\text { R.K. Schumacher, } \\
27 \text { May } 2015\end{array}$ & Frangula alnus & Germany & KY929160 & KY929189 & - & - & - & - \\
\hline & CPC 30973 & $\begin{array}{l}\text { R.K. Schumacher, } \\
10 \text { May } 2016\end{array}$ & $\begin{array}{l}\text { Elaeagnus rhamnoides, } \\
\text { thorn and twig }\end{array}$ & Germany & KY929161 & KY929190 & - & - & - & - \\
\hline & CPC 31482 & $\begin{array}{l}\text { R.K. Schumacher, } \\
22 \text { Jul. } 2016\end{array}$ & Malus domestica, twig & Ukraine & KY929162 & KY929191 & - & - & - & - \\
\hline \multirow[t]{2}{*}{ Querciphoma carteri } & $\begin{array}{l}\text { CBS } 101633=\text { PD } \\
84 / 74\end{array}$ & PD Lisse & Quercus sp. & Netherlands & KF251210 & GQ387593 & KF253166 & - & KF252701 & GQ387532 \\
\hline & CBS 105.91 & H. Schill & $\begin{array}{l}\text { Quercus robur, leaves } \\
\text { and twigs }\end{array}$ & Germany & KF251209 & GQ387594 & KF253165 & - & KF252700 & GQ387533 \\
\hline
\end{tabular}

${ }^{1}$ CBS: Westerdijk Fungal Biodiversity Institute, Utrecht, The Netherlands; CPC: Culture collection of Pedro Crous housed at CBS; INIFAT: Alexander Humboldt Institute for Basic Research in Tropical Agriculture, Ciudad de La Habana, Cuba; PD: Plant Protection Service, nVWA, Division Plant, Wageningen, The Netherlands.

${ }^{2}$ ET: ex-epitype culture; NT: ex-neotype culture; T: ex-type culture.

${ }^{3}$ ITS: internal transcribed spacers and intervening 5.8S nrDNA; LSU: partial 28S nrDNA; SSU: partial 18S nrDNA; tef1: partial translation elongation factor 1-alpha gene; tub2: partial beta-tubulin gene. 
Eurotium chevalieri DAOM 216346 JN938915

- Pseudosydowia eucalypti CPC 14028 GQ303327

68100 Aureobasidium proteae CPC 2824 JN712557

Aureobasidium pullulans CBS 584.75 DQ470956

97 Pseudoseptoria collariana CBS 135104 KF251721

100

- Pseudoseptoria obscura CBS 135103 KF251722

Saccotheciaceae I

Aureobasidiaceae

Saccotheciaceae II

Delphinella strobiligena CBS 735.71 DQ470977

Sydowia polyspora CBS 116.29 DQ678058

- Neocylindroseptoria pistaciae CBS 471.69 KF251656

94 100 Coniozyma leucospermi CBS 111289 EU552113

Endoconidioma populi UAMH 10297 EU981287

99 51 [ Dothidea insculpta CBS 189.58 DQ247802

Dothidea sambuci CBS 197.58 A
Dothidea ribesia CPC 30638

Dothidea ribesia CPC 30713

Dothidea ribesia CPC 30689

Dothidea ribesia CBS 195.58 AY016360

Dothidea puccinioides CBS 193.58 AY004342

64 Dothidea muelleri CBS 191.58 EU167593

$63+$ Dothidea berberidis CBS 187.58 KC800752

Dothidea sp. MFLU 14-0040 KM388552

Dothiora bupleuricola CBS 112.75 KU728538

[ Dothiora agapanthi CPC 20600 KU728537

Dothiora viburnicola CBS 274.72 KU728554

Dothideaceae

Dothiora maculans CBS 299.76 KU728543

- Dothiora sorbi CBS 742.71 KU728552

Dothiora ceratoniae CBS 477.69 KF251655

Dothiora phillyreae CBS 473.69 EU754146

Dothiora oleae CBS 152.71 KU728547

Dothiora cannabinae CBS 737.71 DQ470984

Dothiora laureolae CBS $744.71 \mathrm{KU} 728542$

Dothiora prunorum CBS 933.72 KU728551

Dothiora elliptica CBS $736.71 \mathrm{KU} 728541$

Dothiora phaeosperma CBS 870.71 KU728550

98 Dothiora pyrenophora CPC 30632

Dothiora pyrenophora CPC 30634

Dothiora cactacearum CPC 15585

75 Dothiora cactacearum CPC 15587

Dothiora buxi MFLU 15-3404 KX765295

Pleurophoma acaciae CPC 29188 KY173524

98 Keissleriella cladophila CBS 104.55 GU205221

77_ Keissleriella sparticola MFLUCC 14-0196 KP639571

Keissleriella genistae CBS 113798 GU205222

Pleurophoma ossicola CPC 24985 KR476770

Pleurophoma pleurospora CBS 116668 JF740326

Lentitheciaceae

Keissleriella yonaguniensis KT 2604 AB807594

61. Murilentithecium clematidis IT1078 KM408758

57. Phragmocamarosporium platani MFLUCC 13-0552 KP842916

Phragmocamarosporium hederae MFLUCC 14-1191 KP842915

Didymosphaeria rubi-ulmifolii MFLUCC 14-0023 KJ436586

Paraconiothyrium brasiliense CBS 254.88 JX496171

Pseudocamarosporium sp. 2 CPC 25002

Pseudocamarosporium sp. 2 CPC 25004

Pseudocamarosporium sp. 2 CPC 25843

Pseudocamarosporium sp. 2 CPC 27400

Pseudocamarosporium sp. 2 CPC 30973

Paracamarosporium psoraleae CPC 21632 KF777199

Paracamarosporium fagi CPC 24890 KR611904

53. Paracamarosporium fungicola CBS 113269 JX496133

Paracamarosporium hawailense CBS 120025 DQ885897

Didymosphaeriaceae

Paracamarosporium sp. 1 CPC 30988

10 chānges

Paracamarosporium fagi CPC 31037

Pseudocamarosporium sp. 1 CPC 25926

Pseudocamarosporium brabeji CBS 123026 EU552105

Pseudocamarosporium sp. 2 CPC 31482

Pseudocamarosporium piceae MFLUCC 14-0192 KJ803030

Pseudocamarosporium africanum CBS 121166 JX496142

Fig. 1. One of 1000 equally most parsimonious trees obtained from a maximum parsimony analysis of the LSU sequence alignment. The scale bar shows 10 changes, and parsimony bootstrap support values $>49 \%$ from 1000 replicates are shown at the nodes. Thickened lines represent those branches also present in the strict consensus tree and families are indicated with coloured blocks and the taxonomic novelties, or species treated in the present paper, are in bold text. Orders are indicated on the right side of the tree. The tree was rooted to Eurotium chevalieri (GenBank accession number JN938915). 


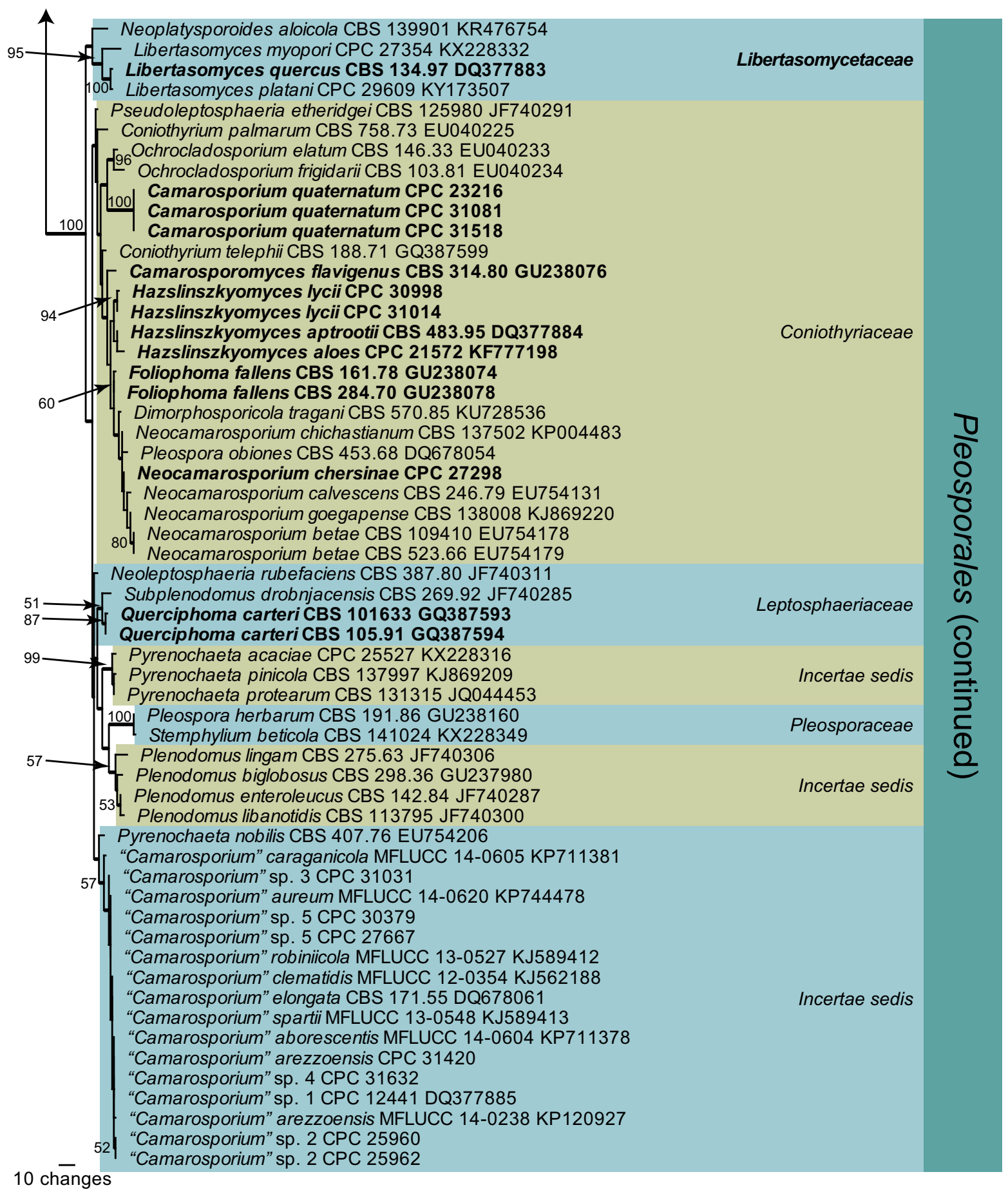

Fig. 1. (Continued).

basally with a few red-brown, smooth, thick-walled and eguttulate hyphae, without setae; ascomatal wall consisting of a textura angularis with red-brown, thick-walled, smooth and eguttulate cells. Pseudoparaphyses numerous, cellular, filiform, multi-celled, short celled, branched, anastomosing, hyaline, smooth, thin-walled, eguttulate, $3.5 \times 4 \mu \mathrm{m}$ diam. Asci 8-spored, cylindrical, apically rounded, pedicel short and furcate, thick-walled, bitunicate, fissitunicate, inamyloid (water plus Lugol), spores oblique uniseriate, 153-172 $\times 16 \times 18.5 \mu \mathrm{m}$. Ascospores (4-)6-celled, ellipsoidal, straight, muriformly septate, golden, faintly thick-walled and smooth, septa in the middle constricted otherwise +/- faintly constricted, mostly the middle cells and seldom both end cells with 1-3 longitudinal septa, eguttulate, without a gelatinous sheath and appendages, rehydrated and examined in water, mature (20-)22.5(-26.5) × (10-)11.5(13) $\mu \mathrm{m},(1.79-) 1.99(-2.2)(\mathrm{l} / \mathrm{b})$. Conidiomata pycnidial, saprobic, subcorticolous, single to gregarious, partly caespitose, globose seldom broad pyroid with a flattened base, ostiole centrally, terete, short papillate, and somewhat erumpent, black, soft, +/-thin, covered with hyaline to ochre and smooth hyphae, to $0.8 \mathrm{~mm}$ diam; conidiomatal wall few-layered, consisting of a textura globulosa-angularis with red brown, thick-walled, and smooth cells. Paraphyses 
Dothiora oleae CBS 615.72 KU728511.1

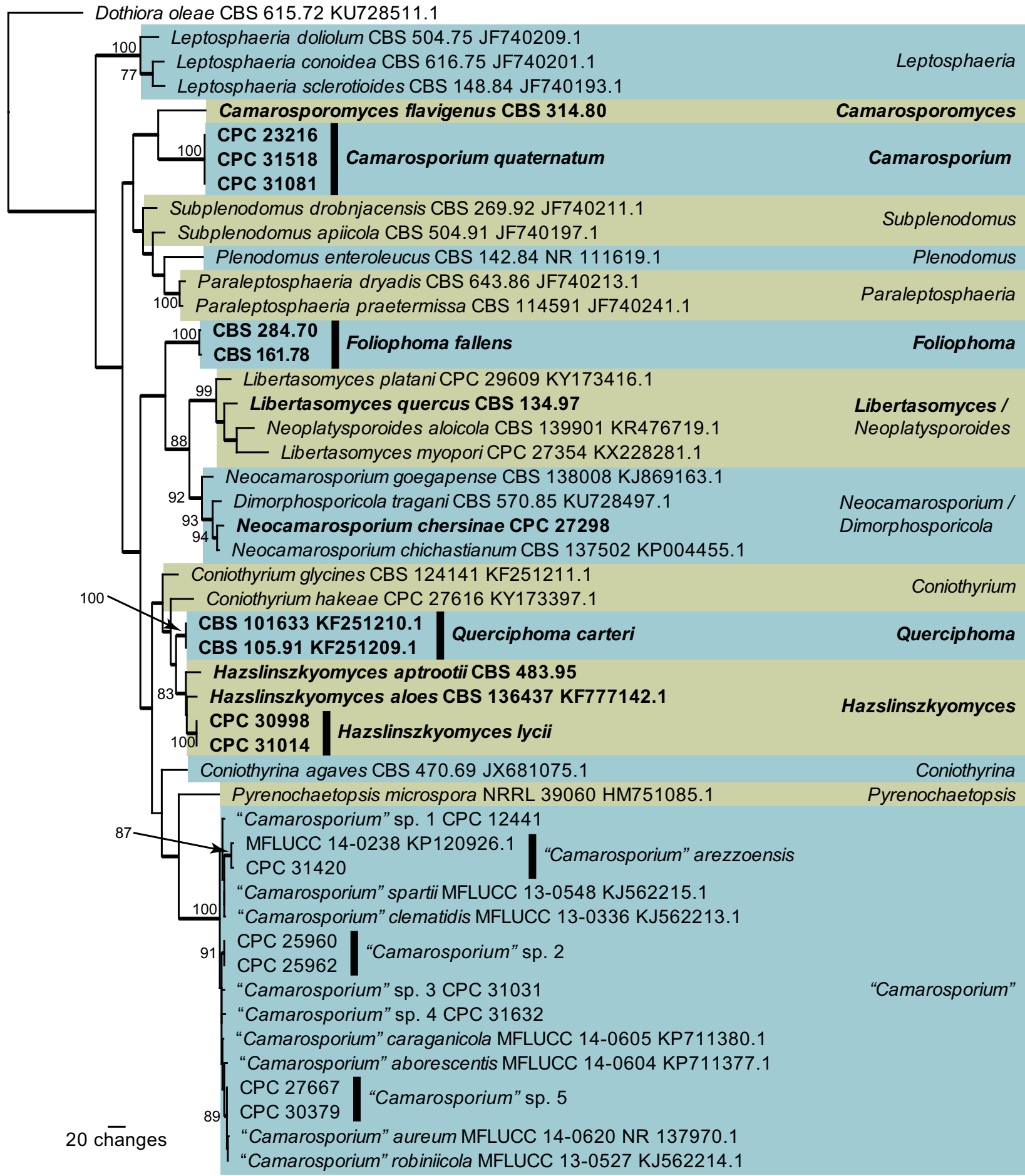

Fig. 2. One of 284 equally most parsimonious trees obtained from a maximum parsimony analysis of the ITS sequence alignment of Camarosporium and allied genera. The scale bar shows 20 changes, and parsimony bootstrap support values $>74 \%$ from 1000 replicates are shown at the nodes. Thickened lines represent those branches also present in the strict consensus tree and genera are indicated with coloured blocks and the taxonomic novelties, or species treated in the present paper, are in bold text. The tree was rooted to Dothiora oleae (GenBank accession number KU728511).

and conidiophores absent. Conidiogenous cells formed from the inner cells of the pycnidial wall, doliiform, hyaline, thin-walled, smooth, eguttulate, annellidic, secession apically and singly. Conidia as brownish, dark olive to black drops in mass, (3-)4(-6)-celled, muriformly septate, with one longitudinal or diagonal septum per cell and 1-2 per conidium, ellipsoidal, pyroid, clavate, straight to slightly curved, apically rounded, base often tapered but never truncate, always yellowish, but never brown, both end cells but mostly the basal cell often paler or hyaline, wall golden, thin and smooth, septa golden, thick-walled and smooth to slightly constricted, eguttulate at maturity, examined in water, living and mature, (18-)26.5(-34) × (9-)12(-16) $\mu \mathrm{m},(1.19-) 2.26(-3.32)(\mathrm{l} / \mathrm{b})$. Synasexual morph in culture: 
Pezicula cinnamomea CBS 290.39 KR859133.1

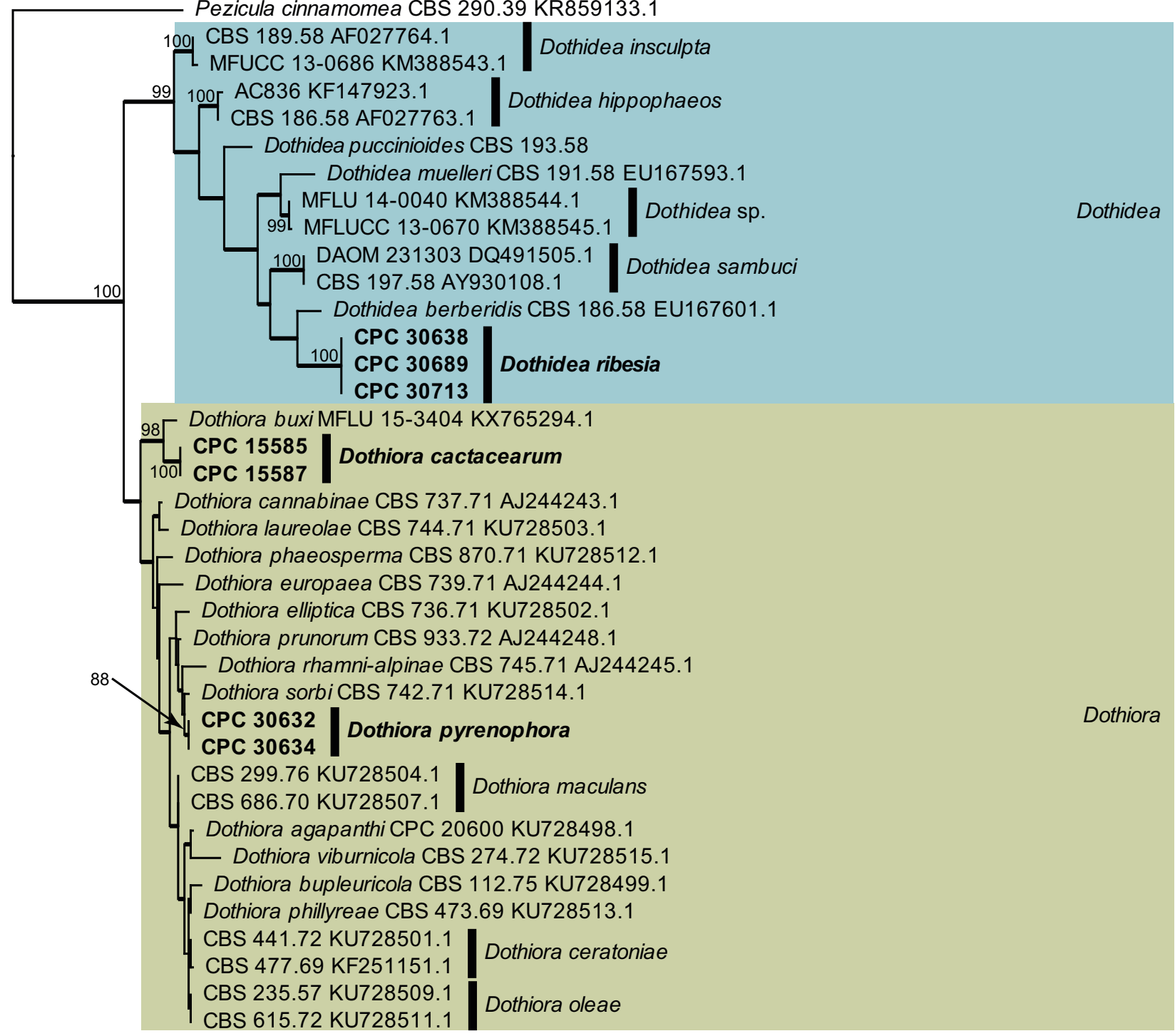

10 changes

Fig. 3. One of 140 equally most parsimonious trees obtained from a maximum parsimony analysis of the ITS sequence alignment of Dothidea and Dothiora. The scale bar shows 10 changes, and parsimony bootstrap support values $>74 \%$ from 1000 replicates are shown at the nodes. Thickened lines represent those branches also present in the strict consensus tree and genera are indicated with coloured blocks and the taxonomic novelties, or species treated in the present paper, are in bold text. The tree was rooted to Pezicula cinnamomea (GenBank accession number KR859133).

Conidiomata separate, pycnidial, immersed to superficial on PNA, brown, globose, 150-200 $\mu \mathrm{m}$ diam, with 1-2 papillate ostioles, exuding a crystalline conidial mass. Conidiophores reduced to conidiogenous cells. Conidiogenous cells lining the inner cavity, hyaline, smooth, ampulliform, with periclinal thickening at apex, 3-5 × 4-5 $\mu \mathrm{m}$. Conidia solitary, hyaline, smooth, aseptate, subcylindrical, straight, rarely curved, apex obtuse, base truncate, (3-)4-5(-6) × $1.5 \mu \mathrm{m}$.

Culture characteristics: On MEA spreading, with fluffy aerial mycelium and feathery margin; surface dirty white with patches of olivaceous grey. On PDA surface and reverse olivaceous grey. On OA surface olivaceous grey with patches of dirty white and umber.
Notes: Single conidial colonies of $C$. quaternatum formed a phoma-like fungus in culture. The single conidial isolation step was redone three times on different media, with the same result. It was only once colonies were subcultured onto SNA plates supplemented with autoclaved pieces of banana leaf, that a few typical Camarosporium conidiomata again developed on leaf pieces. The trigger influencing which morph develops in culture, however, remains unknown, with the phoma-like morph being most commonly encountered, and the Camarosporium morph extremely rare.

Ascospores of Camarosporium quaternatum studied here agree with those of the original description of Cucurbitaria varians (figs 13-24), and conidia that of its asexual morph, Clinterium quaternatum (figs 9-12) (Hazslinszky 1865). Because the holotype material could not be traced, the original illustrations are designated as lectotypes, to facilitate epitypification. 
Keissleriella trichophoricola CBS 136770 KJ869113.1

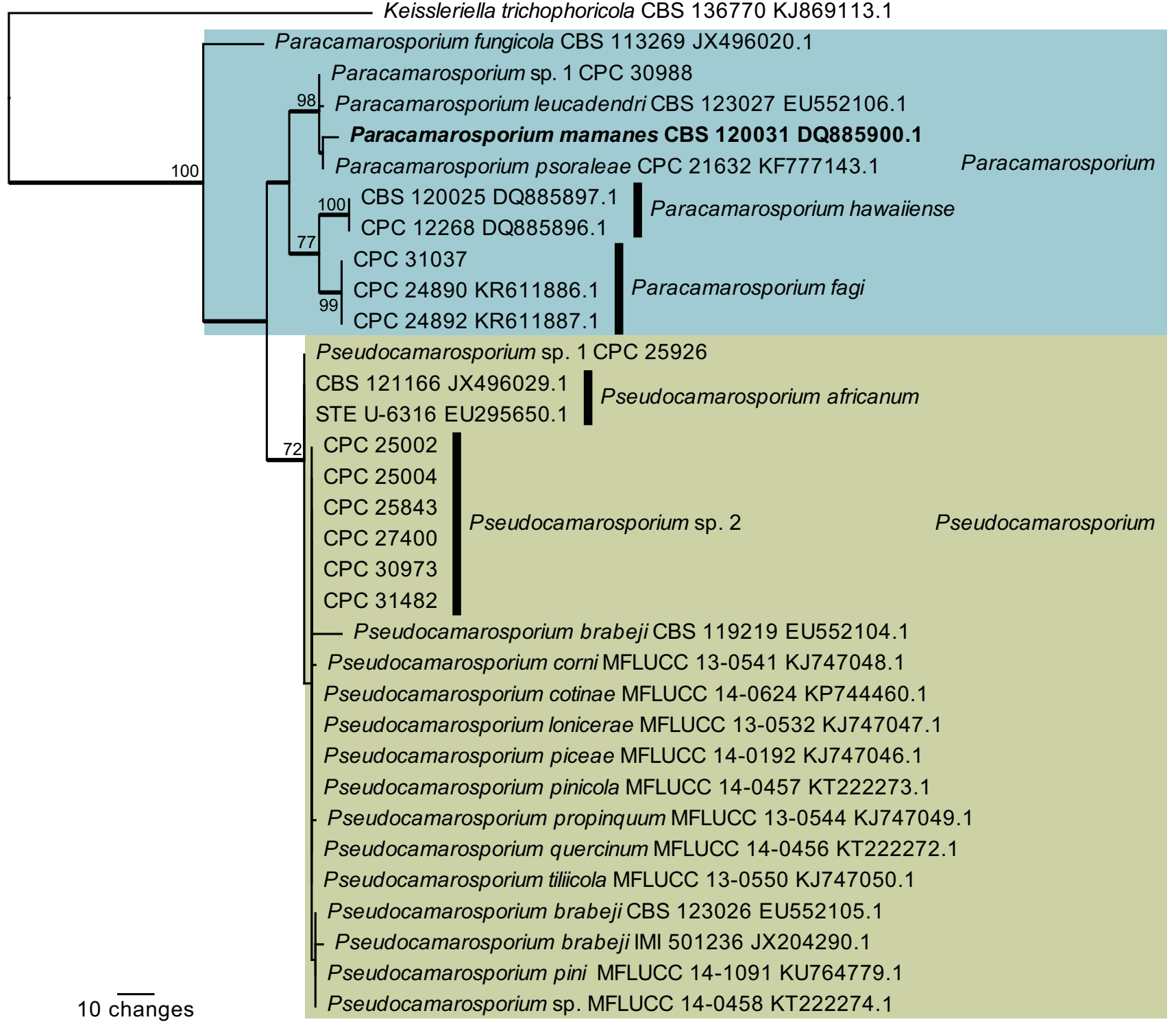

Fig. 4. One of 16 equally most parsimonious trees obtained from a maximum parsimony analysis of the ITS sequence alignment of Paracamarosporium and Pseudocamarosporium. The scale bar shows 10 changes, and parsimony bootstrap support values $>69 \%$ from 1000 replicates are shown at the nodes. Thickened lines represent those branches also present in the strict consensus tree and genera are indicated with coloured blocks and the taxonomic novelties, or species treated in the present paper, are in bold text. The tree was rooted to Keissleriella trichophoricola (GenBank accession number KJ869113).

Table 2. Statistical information on the individual alignments and number of equally most parsimonious trees saved for each dataset analysed ${ }^{1}$.

\begin{tabular}{lllll}
\hline & LSU overview & Camarosporium ITS & Dothidea ITS & Paracamarosporium ITS \\
\hline \hline Aligned characters (including gaps) & 722 & 554 & 530 & 523 \\
Parsimony-informative characters & 192 & 242 & 109 & 45 \\
Variable and parsimony-uninformative characters & 55 & 88 & 107 & 143 \\
Constant characters & 475 & 224 & 314 & 335 \\
Equally most parsimonious trees obtained & 1000 & 284 & 140 & 16 \\
Tree length & 673 & 1247 & 470 & 249 \\
Consistency index (Cl) & 0,551 & 0,499 & 0,691 & 0,912 \\
Retention index (RI) & 0,948 & 0,764 & 0,832 & 0,891 \\
Rescaled Consistency index (RC) & 0,522 & 0,381 & 0,575 & 0,812 \\
\hline
\end{tabular}

1 ITS: internal transcribed spacers and intervening 5.8S nrDNA; LSU: partial $28 \mathrm{~S}$ nrDNA. 

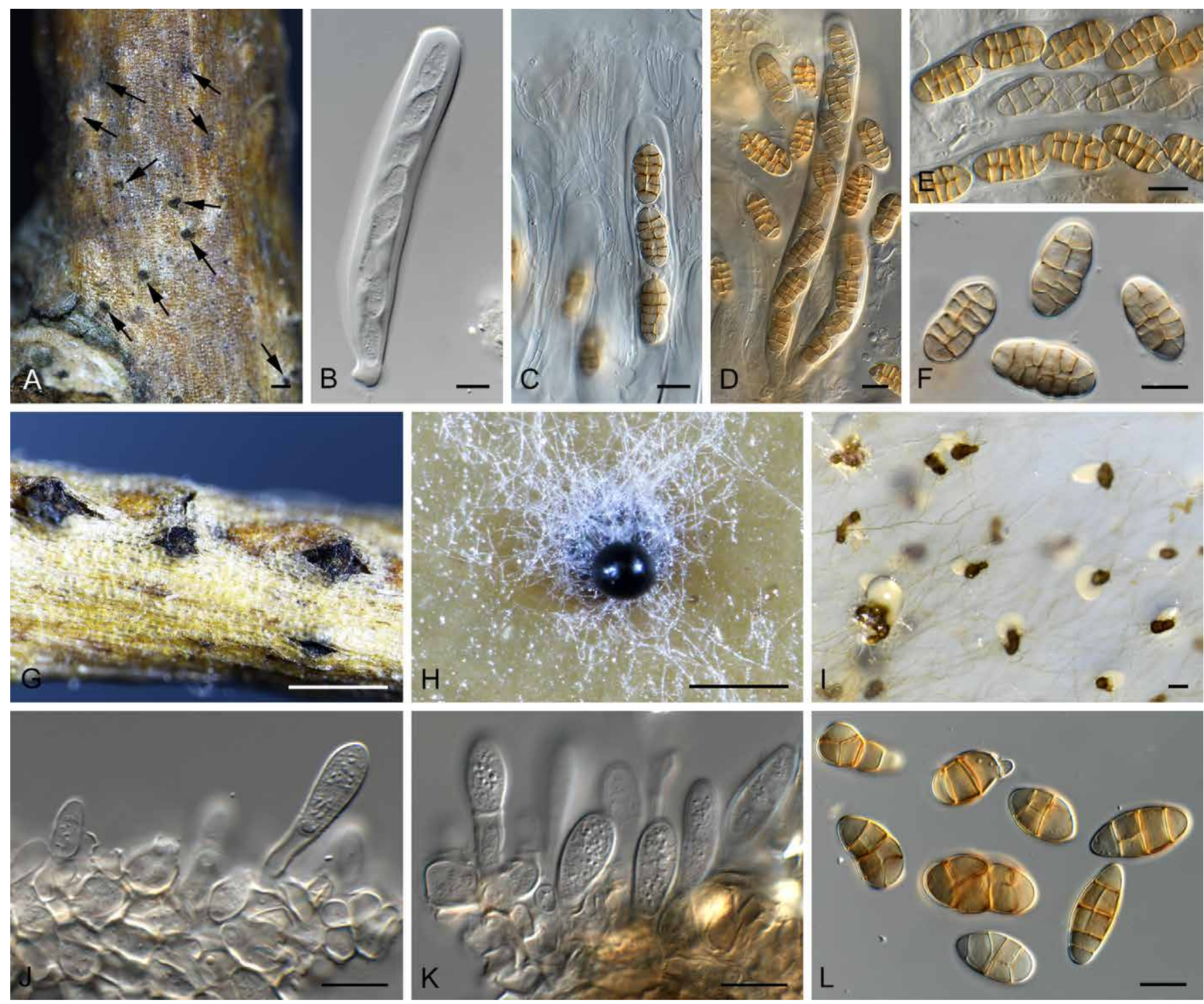

Fig. 5. Camarosporium quaternatum (CPC 31081). A. Immersed ascomata on twig (arrows). B-E. Asci and pseudoparaphyses. F. Ascospores.

G. Conidiomata on twig. H. Camarosporium conidioma on OA. I. Phoma-like conidiomata on SNA. J-K. Conidiogenous cells giving rise to Camarosporium conidia. L. Conidia. Bars: $\mathrm{A}, \mathrm{I}=200 \mu \mathrm{m}, \mathrm{G}=800 \mu \mathrm{m}, \mathrm{H}=300 \mu \mathrm{m}$, all others $=10 \mu \mathrm{m}$.

Saccardo (1883) reported the fungus to occur on Lycium barbarum in Hungary and Germany. In the present study, we also found the sexual morph on Daphne mezereum, a new host for $C$. quaternatum. The two cultures used in previous studies as representative of this fungus (CBS 483.95, CBS 134.97) (e.g. Crous et al. 2006) represent two camarosporium-like fungi that are morphologically distinct from C. quaternatum.

Sutton (1980) stated that the genus Camarosporium, which contains several hundred species, is polyphyletic and in need of revision. As we have shown here, Camarosporium s. str. is distinct from other taxa that have been treated as representative of or similar in some respects to Camarosporium, viz. Henfellra, Neocamarosporium, Paracamarosporium, or Xenocamarosporium (Crous et al. 2013, 2014b, 2015b, Wijayawardene et al. 2014, 2016, Hawksworth et al. 2016). Further studies are underway to treat those taxa that are presently known from culture.

During the course of evaluating the status of Camaro- sporium s. str., several other camarosporium-like or pleospora-like isolates also had to be examined and were found to be undescribed. These are treated below:

\section{Camarosporomyces Crous, gen. nov. MycoBank MB820901}

Etymology: Named after its morphological similarity to Camarosporium and its phoma-like synasexual morph.

Diagnosis: Distinct from phoma-like genera in having pycnidial conidiomata with prominent papillate dark brown periphysate ostiole, and thicker wall at apex.

Type species: Camarosporomyces flavigenus (Constantinou \& Aa) Crous 2017.

Description: Conidiomata pycnidial, solitary to aggregated, obovoid, medium brown, with prominent papillate ostiole, 

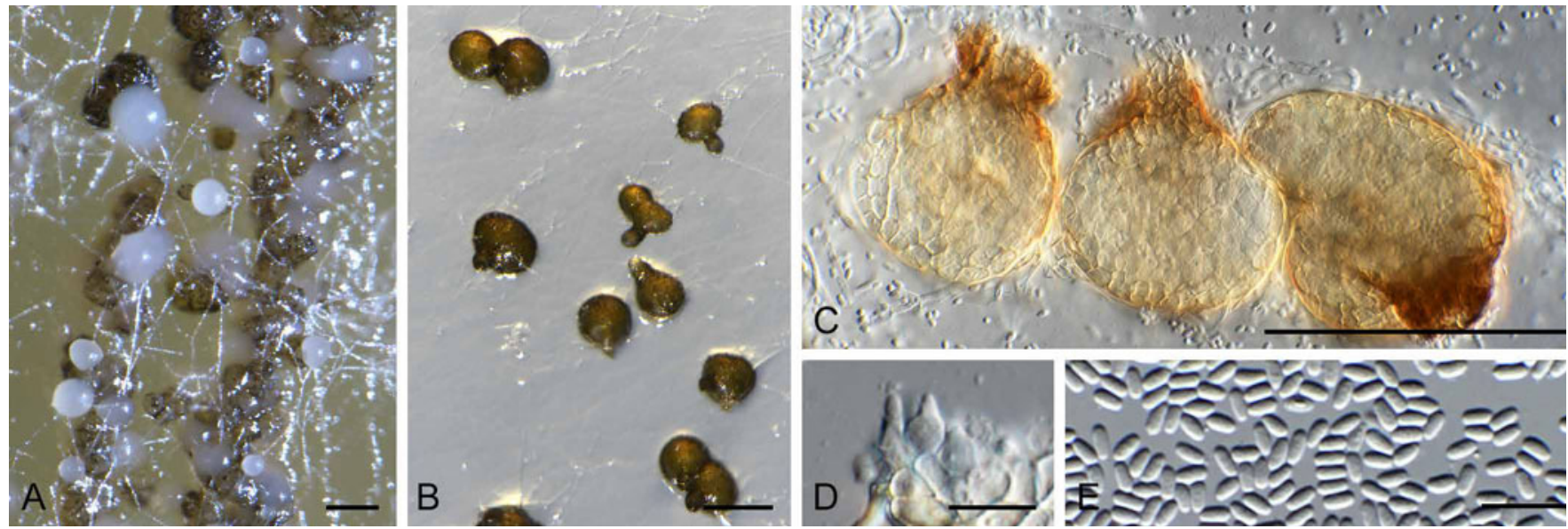

Fig. 6. Camarosporomyces flavigenus (CBS 314.80). A. Conidiomata on PDA. B. Conidiomata on SNA. C. Conidiomata showing darker ostiolar area. D. Conidiogenous cells. E. Phoma-like conidia. Bars: A-C $=90 \mu \mathrm{m}, \mathrm{D}-\mathrm{E}=10 \mu \mathrm{m}$.

dark brown, with extra 1-2 layers of wall at the apex (thicker than pycnidial body), periphysate. Conidiophores reduced to conidiogenous cells lining inner cavity, hyaline, smooth, ampulliform; phialidic with periclinal thickening. Conidia solitary, hyaline, smooth, aseptate, subcylindrical, straight, apex obtuse, base truncate.

\section{Camarosporomyces flavigenus (Constant. \& Aa) Crous, comb. nov. \\ MycoBank MB820902}

(Fig. 6)

Basionym: Phoma flavigena Constant. \& Aa, Trans. Brit. Mycol. Soc. 79: 343 (1982).

Synonym: Pleospora flavigena (Constant. \& Aa) Gruyter \& Verkley, Stud. Mycol. 75: 25 (2013) ["2012"].

Type: Romania: Bucharest, station for water treatment, from water, 1980, K. Fodor (CBS H-23067 - holotype; CBS 314.80 - culture ex-type).

Description: Conidiomata pycnidial, solitary to aggregated, obovoid, medium brown, 50-90 $\mu \mathrm{m}$ diam, with prominent papillate ostiole, dark brown, 10-25 $\mu \mathrm{m}$ diam with extra 1-2 layers of wall at the apex (thicker than pycnidial body), periphysate. Conidiophores reduced to conidiogenous cells lining inner cavity, hyaline, smooth, ampulliform, 4-5 × 3-4 $\mu \mathrm{m}$; phialidic with periclinal thickening. Conidia solitary, hyaline, smooth, aseptate, subcylindrical, straight, apex obtuse, base truncate, $(2.5-) 3 \times 1.5 \mu \mathrm{m}$.

Culture characteristics: Colonies flat, spreading, with sparse aerial mycelium, margins smooth, lobate, reaching $30 \mathrm{~mm}$ diam after 2 wk. On OA surface umber with diffuse yellow pigment. On PDA surface umber, outer region pale luteous, reverse umber.

Note: Camarosporomyces flavigenus is a phoma-like fungus which was originally described as Phoma flavigena, and later placed in Pleospora by De Gruyter et al. (2013), who used a much wider circumscription of the genus Pleospora than applied here.
Foliophoma Crous, gen. nov. MycoBank MB820903

Etymology: Named after its association with leaf spots, and its morphological similarity to Phoma.

Diagnosis: Distinct from phoma-like genera in having eustromatic conidiomata, uni- to multi-loculate with 1-3 ostioles. Conidiogenous cells with periclinal thickening or percurrent proliferation at apex.

Type species: Foliophoma fallens (Sacc.) Crous 2017.

Description: Conidiomata globose, eustromatic, uni- to multi-locular with 1-3 ostioles, medium brown, outer surface smooth; wall of 3-6 layers of brown textura angularis. Conidiophores reduced to conidiogenous cells lining inner cavity, hyaline, smooth, doliiform to subcylindrical; phialidic with periclinal thickening or percurrent proliferation at apex. Conidia aseptate, solitary, hyaline, smooth, guttulate to granular, broadly ellipsoidal, thick-walled, apex obtuse, base truncate to bluntly rounded.

Foliophoma fallens (Sacc.) Crous, comb. nov. MycoBank MB820904

(Fig. 7)

Basionym: Phoma fallens Sacc., Syll. Fung. 10: 146 (1892).

Synonyms: Pleospora fallens (Sacc.) Gruyter \& Verkley, Stud. Mycol. 75: 25 (2013) ["2012"].

Phyllosticta glaucispora Delacr., Bull. Soc. Mycol. France 9: 266 (1893).

Phoma glaucispora (Delacr.) Noordel. \& Boerema, Versl. Meded. Plantenziektenk. Dienst Wageningen 166 : 108 (1989) [“1988"].

Phyllosticta oleandri Gutner, Trudy Bot. Inst. Akad. Nauk S.S.S.R., ser. 2, Sporov. Rast. 1: 306 (1933).

Description: Conidiomata globose, eustromatic, uni- to multilocular with 1-3 ostioles, 120-250 $\mu \mathrm{m}$ diam, medium brown, outer surface smooth; wall of 3-6 layers of brown textura angularis. Conidiophores reduced to conidiogenous cells lining inner cavity, hyaline, smooth, doliiform to subcylindrical, 

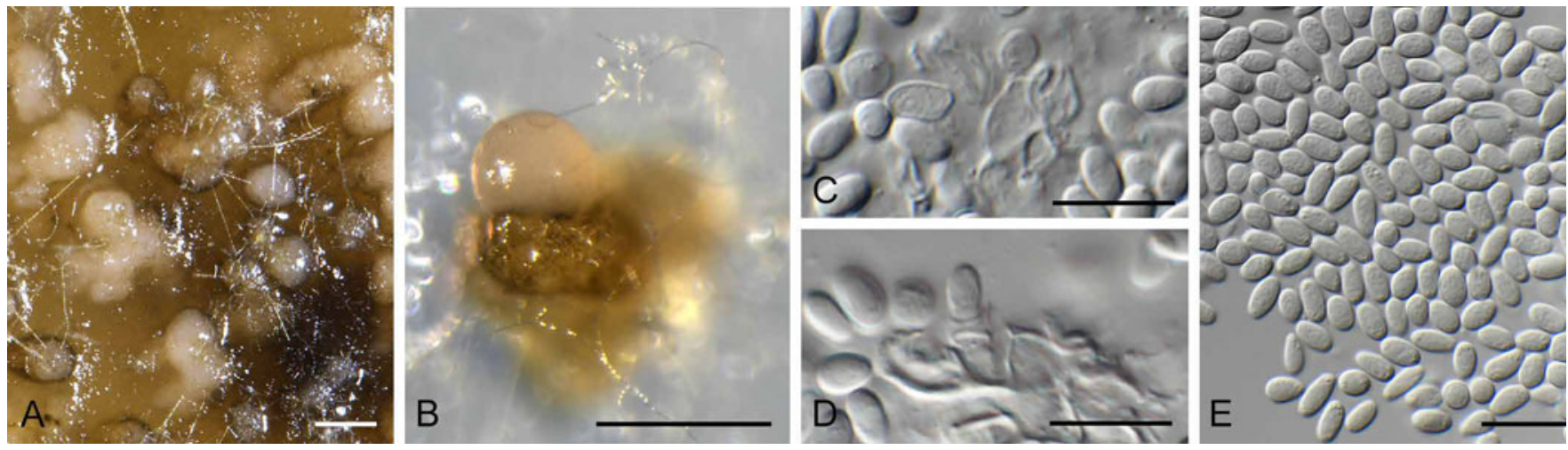

Fig. 7. Foliophoma fallens (CBS 284.70). A. Conidiomata on PDA. B. Conidiomata on SNA. C-D. Conidiogenous cells. E. Conidia. Bars: A-B $=250 \mu \mathrm{m}$, all others $=10 \mu \mathrm{m}$.

5-7 $\times 4-5 \mu \mathrm{m}$; phialidic with periclinal thickening or percurrent proliferation at apex. Conidia aseptate, solitary, hyaline, smooth, guttulate to granular, broadly ellipsoidal, thick-walled, apex obtuse, base truncate to bluntly rounded, (5-)5.5-6(-7) $\times(3-) 4(-5) \mu \mathrm{m}$ (based on CBS 284.70).

Culture characteristics: Colonies flat, spreading, with moderate aerial mycelium and even, lobate margins, reaching $50 \mathrm{~mm}$ diam after $2 \mathrm{wk}$. On PDA surface sepia to umber, reverse dark mouse grey. On OA surface greyish sepia.

Material examined: Italy: Capri, Villa Jovis, leaf spot on Nerium oleander (Apocynaceae), Apr. 1970, H.A. van der Aa (CBS H-16639, 23066, culture CBS 284.70). - New Zealand: Levin, from leaf spot of Olea europaea (Oleaceae), 1978, G.F. Laundon (CBS $161.78=$ LEV 1131).

Notes: Phoma fallens is associated with leaf spots of Olea europaea in Europe (conidia 7-9 × 3-4 $\mu \mathrm{m}$ ), while Phoma glaucispora (conidia 5-8 $\times 2.5-4.5 \mu \mathrm{m}$ ) is associated with leaf spots on Nerium oleander in Europe (Boerema et al. 2004). Phylogenetically, however, De Gruyter et al. (2013) found these two species to be closely related, and therefore treated them as a single species, placing them in Pleospora based on the larger circumscription used at the time. However, the present fungus is not congeneric with Stemphylium herbarum (syn. Pleospora herbarum; Rossman et al. 2015), and therefore a new generic name is introduced to accommodate it.

\section{Hazslinszkyomyces Crous \& R.K. Schumach., gen. nov.}

MycoBank MB820905

Etymology: Named after Friedrich August Hazslinszky von Hazslin (1818-1896), in recognition for his work on camarosporium-like fungi.

Diagnosis: Morphologically similar to Camarosporium, but distinct as conidia are uniformly brown in colour, those of Camarosporium having paler end cells.

Type species: Hazslinszkyomyces aloes (Crous \& M.J. Wingf.) Crous 2017.
Description: Ascomata pseudothecial, saprobic, subcorticolous, densely crowded, erumpent, globose to pyroid with a flattened base, ostiole central, terete, and short papillate, black, periphysate; ascomatal wall multi-layered, consisting of a textura angularis. Pseudoparaphyses numerous, basally moniliform, upwards filiform, multi-celled, hyaline, thin-walled, smooth. Asci 8-spored, cylindrical, thick-walled, apically rounded and with an ocular chamber, pedicel short and furcate, bitunicate, fissitunicate. Ascospores transversely septate, constricted at the median septum, becoming muriformly septate, ellipsoidal, golden brown. Phoma-like morph: Conidiomata pycnidial, solitary, brown, globose, with central ostiole; wall of 2-3 layers of brown textura angularis. Conidiophores reduced to conidiogenous cells lining the inner cavity, hyaline, smooth, doliiform to subcylindrical. Conidia solitary, hyaline, smooth, guttulate, subcylindrical, straight, aseptate. Camarosporium-like morph: Occurring in the same or separate conidioma with a phoma-like morph. Conidiogenous cells lining the inner cavity, doliiform to subcylindrical, hyaline, smooth, proliferating percurrently at apex. Conidia solitary, brown, smooth, broadly ellipsoidal to obovoid, transversely septate, becoming muriformly septate.

\section{Hazslinszkyomyces aloes (Crous \& M.J. Wingf.)} Crous, comb. nov.

MycoBank MB820906

Basionym: Camarosporium aloes Crous \& M.J. Wingf., Persoonia 31: 247 (2013).

Type: South Africa: Western Cape Province: Clanwilliam, on dark lesions on dead bark of Aloe dichotoma (Xanthorrhoeaceae), Sept. 2012, M.J. Wingfield (CBS $\mathrm{H}-21446$ - holotype; CPC 21572 = CBS 136437 - culture ex-type).

\section{Hazslinszkyomyces aptrootii Crous, sp. nov.} MycoBank MB820908

(Fig. 8)

Etymology: Named after André Aptroot, who collected this fungus in The Netherlands. 

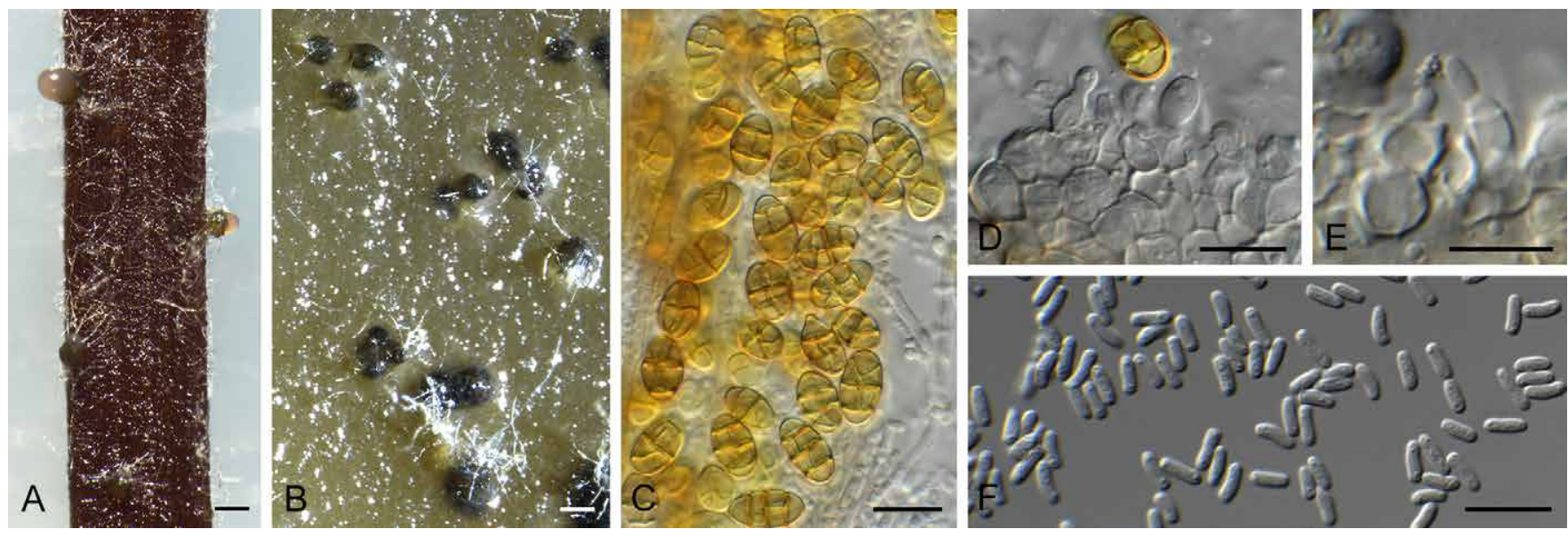

Fig. 8. Hazslinszkyomyces aptrootii (CBS 483.95). A. Conidiomata on PNA. B. Conidiomata on PDA. C. Hazslinszkyomyces and phoma-like conidia. D-E. Conidiogenous cells. F. Phoma-like conidia. Bars: A-B $=170 \mu \mathrm{m}$, all others $=10 \mu \mathrm{m}$.

Diagnosis: Similar to Hazslinszkyomyces aloes [conidia (9-) $11-13(-14) \times(4-) 6-7(-8) \mu \mathrm{m}]$, but distinct in having larger conidia.

Type: The Netherlands: Province of Noord-Holland: Egmond, on Lycium sp., 17 Mar. 1995, A. Aptroot (CBS H-23068 holotype; CBS 483.95 - culture ex-type).

Description: Conidiomata separate, erumpent, pycnidial, globose, brown, 130-170 $\mu \mathrm{m}$ diam, with central, non-papillate ostiole, $10 \mu \mathrm{m}$ diam, exuding a creamy conidial mass; wall of 3-6 layers of brown textura angularis. Conidia dimorphic. Phoma-like morph: Conidiophores reduced to conidiogenous cells lining the inner cavity, ampulliform to subcylindrical, hyaline, smooth, phialidic with periclinal thickening at apex, becoming percurrent with age, 5-7 × 3-6 $\mu \mathrm{m}$. Conidia solitary, hyaline, smooth, guttulate, subcylindrical, aseptate, straight to curved, apex obtuse, base truncate, (4-)5-6 $\times$ $2 \mu \mathrm{m}$. Camarosporium-like morph: Occurring in the same conidiomata as the phoma-like morph. Conidiophores reduced to conidiogenous cells, hyaline, smooth, doliiform to subcylindrical, proliferating percurrently at apex, 5-7 × 4-6 $\mu \mathrm{m}$. Conidia solitary, broadly ellipsoidal, apex subobtusely rounded, base truncate, hilum $2 \mu \mathrm{m}$ diam, medium brown, smooth, muriformly septate, with 3 transverse septa, and 1-3 oblique septa, (11-)13-14(-16) × (7-)8(-9) $\mu \mathrm{m}$.

Culture characteristics: Colonies flat, spreading, with sparse to moderate aerial mycelium, covering dish in $2 \mathrm{wk}$, with even, lobate margins. On PDA surface umber, reverse brown vinaceous. On OA surface chestnut to brown vinaceous.

Notes: This collection was originally identified as Camarosporium quaternatum, based on its general morphology, and the fact that it occurred on Lycium. Morphologically, however, the conidia are much smaller than those of $C$. quaternatum, and thus it is described as a new species of Hazslinszkyomyces. Phylogenetically the two genera are also quite distinct.

\section{Hazslinszkyomyces lycii Crous \& R.K. Schumach., sp. nov.}

MycoBank MB820909

(Fig. 9)

Etymology: Named after the host genus from which it was isolated, Lycium.

Diagnosis: Similar to Hazslinszkyomyces aptrootii [conidia $(11-) 13-14(-16) \times(7-) 8(-9) \mu \mathrm{m}]$, but distinct in having larger conidia.

Type: Hungary: near Budapest, on twig of Lycium barbarum (Solanaceae), 2 May 2016, L. Bartalos (CBS H-23069 holotype; CPC 30998 = CBS 142619 - culture ex-type).

Description: Ascomata pseudothecial, saprobic, subcorticolous, densely crowded, erumpent, globose to pyroid with a flattened base, ostiole central, terete, and short papillate, black, soft, thick, to $130 \mu \mathrm{m}$ diam, periphysate; ascomatal wall multi-layered, consisting of a textura angularis with red-brown, thick-walled, smooth and small cells. Pseudoparaphyses numerous, basally moniliform, upwards filiform, multi-celled, short-celled, branched, with anastomoses, gnarled, hyaline, thin-walled, smooth. Asci 8-spored, cylindrical, thick-walled, apically rounded and with an ocular chamber, 2-3 $\mu \mathrm{m}$ diam, pedicel short and furcate, bitunicate, fissitunicate, 185-214 $\times$ $14.5 \times 15.5 \mu \mathrm{m}$, ascospores oblique uniseriate. Ascospores initially with three transverse septa, constricted at the median septum, developing up to five transverse septa, becoming muriformly septate, 1-2 longitudinal or diagonal septa per cell, ellipsoidal, straight, the upper part often wider, end cells mostly bluntly rounded seldom conical, wall faintly thick and smooth, golden brown, septa red-golden and faintly thick, median septum constricted otherwise faintly constricted, eguttulate at maturity, no mucilaginous sheath in all stages of development, in lactic acid (18-)20-21(-23) × (8.5-)9-10(12) $\mu \mathrm{m}$, in water $20.5-30.5 \times 10-15 \mu \mathrm{m}$. Phoma-like morph: Conidiomata pycnidial, solitary, brown, globose, 180-250 $\mu \mathrm{m}$ diam, with central ostiole, 20-30 $\mu \mathrm{m}$ diam; wall of 2-3 layers of brown textura angularis. Conidiophores reduced to conidiogenous cells lining the inner cavity, hyaline, 

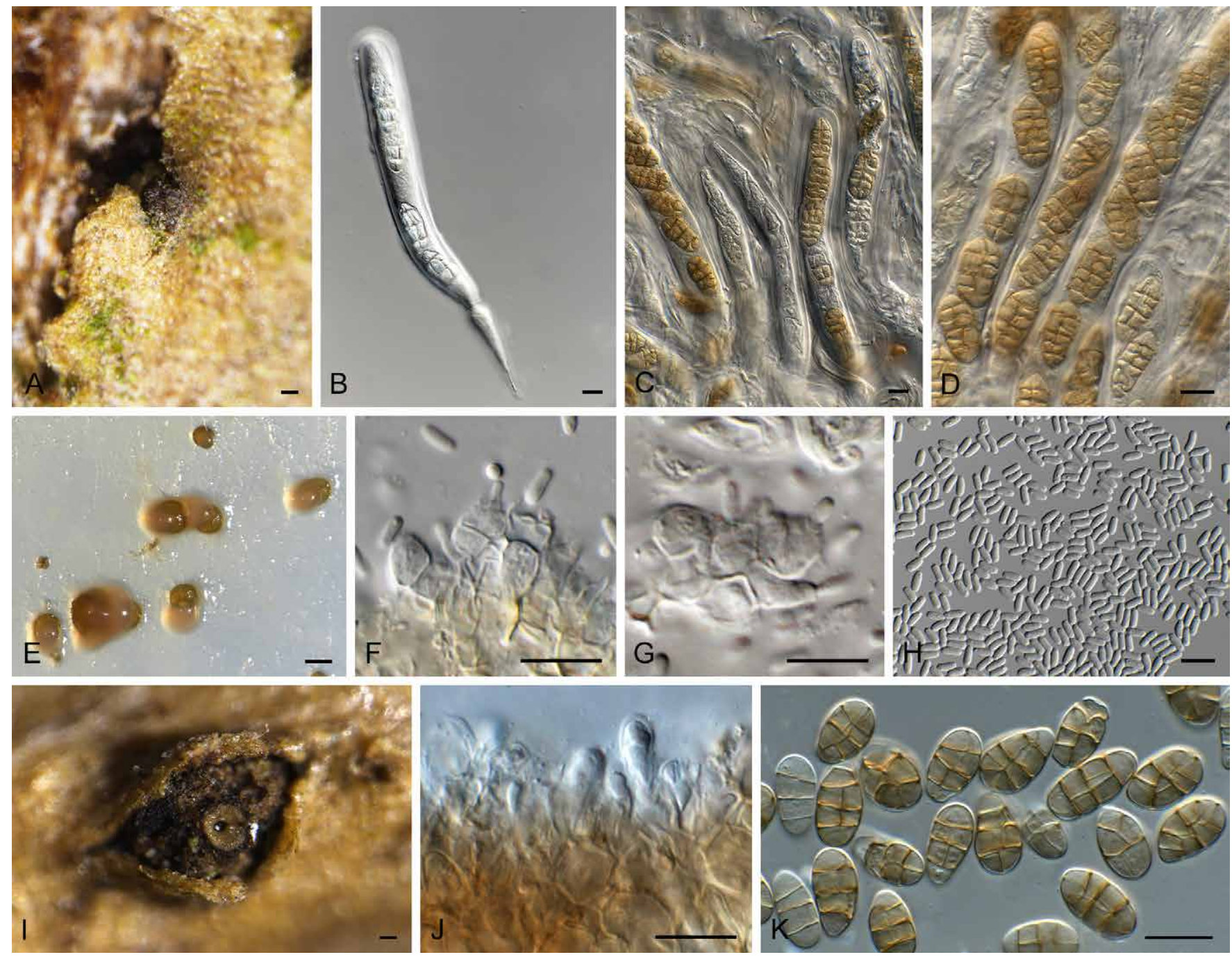

Fig. 9. Hazslinszkyomyces lycii (CPC 30998). A. Ascoma immersed in twig. B-D. Asci and pseudoparaphyses. E. Phoma-like conidiomata on SNA. F-G. Conidiogenous cells. H. Conidia. I. Hazslinszkyomyces conidioma on twig. J. Conidiogenous cells. K. Conidia. Bars: A = $130 \mu$ m, I $=250 \mu \mathrm{m}$, all others $=10 \mu \mathrm{m}$.

smooth, doliiform to subcylindrical, 4-7 $\times 4-5 \mu \mathrm{m}$; phialidic, with periclinal thickening at apex. Conidia solitary, hyaline, smooth, guttulate, subcylindrical, straight, aseptate, apex obtuse, base truncate, hilum $1.5 \mu \mathrm{m}$ diam, $(3.5-) 4-5(-6) \times$ $2 \mu \mathrm{m}$. Camarosporium-like morph: Conidiomata pycnidial, solitary, gregarious, brown, globose, with central ostiole, wall few-layered of brown textura globulosa-angularis, completely covered with hyaline to brownish and short hyphae, to $800 \mu \mathrm{m}$ diam. Conidiogenous cells lining the inner cavity, doliiform to subcylindrical, hyaline, smooth, 6-7 × 5-7 $\mu \mathrm{m}$; annellidic, proliferating percurrently at apex. Conidia solitary, pale yellowish with a golden wall, smooth, broadly ellipsoidal to obovoid but also clavate, seldom cylindrical, straight, seldom slightly curved, apex obtuse, base sometimes tapered and never truncate, 4-5 $\mu \mathrm{m}$ diam, transversely (2-)3(-4-5)-septate, becoming muriformly septate, with vertical and oblique septa, septa smooth to slightly constricted and thick, eguttulate, (15-)16-18(-20) $\times(8-) 9(-10) \mu \mathrm{m}$ in vitro, 18-34 × 9-16 $\mu \mathrm{m}$ in vivo.

Culture characteristics: Colonies covering dish in $2 \mathrm{wk}$, with moderate aerial mycelium, and smooth, lobate margins. On
PDA surface fawn to hazel, reverse fawn. On OA surface ochreous to umber.

Other material examined: Hungary: near Budapest, on twig of Lycium barbarum (Solanaceae), 1 May 2016, L. Bartalos (HPC 1029 $=$ CPC $31014=$ CBS 142618).

Notes: Hazslinszkyomyces lycii occurred in association with a camarosporium-like morph which, when cultured, proved to be identical based on DNA sequence data. The sexual morph also produced a phoma-like synasexual morph in culture. Pyrenophora lycii (syn. Pleospora lycii), also described from Lycium barbarum in Hungary, has setose pseudothecia and ascospores that are transversely 5-septate with only one longitudinal septum per cell, 26-30 $\times 8 \mu \mathrm{m}$, and is distinct from the present fungus. A further name to consider is "Hendersonia lycii Hazsl". Hazslinszky (1865) referred to a Hendersonia form on Lycium barbarum, but the name Hendersonia lycii was never validly published with any description, and has crept into literature in error, and is thus unavailable for this collection. The taxon is therefore described here as new. 

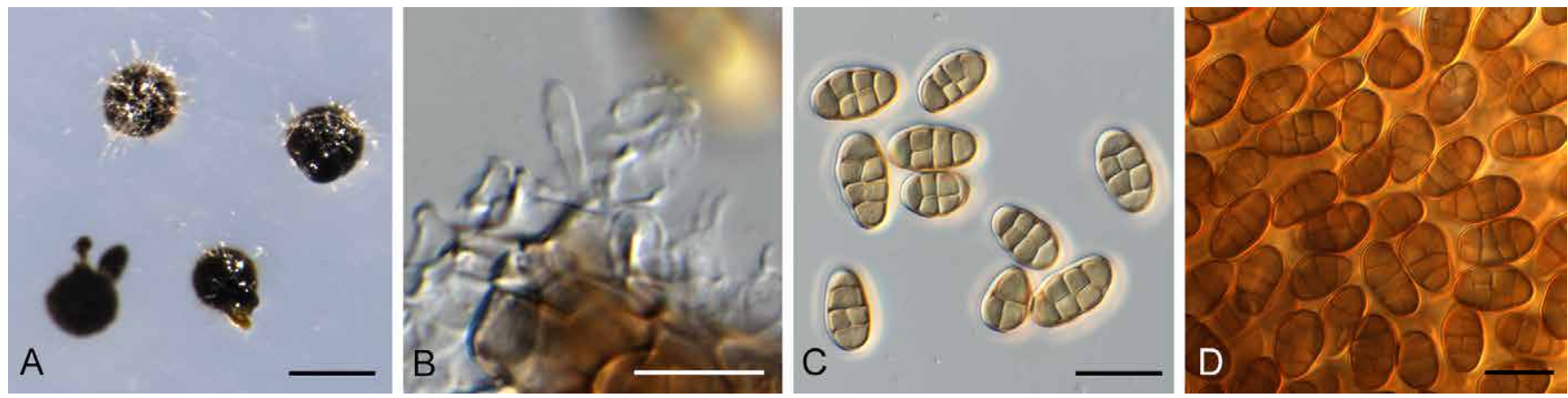

Fig. 10. Neocamarosporium chersinae (CPC 27298). A. Conidiomata on SNA. B. Conidiogenous cells. C-D. Conidia. Bars: $A=300 \mu m$, all others $=10 \mu \mathrm{m}$.

\section{Neocamarosporium chersinae Crous, sp. nov. MycoBank MB820910 \\ (Fig. 10)}

Etymology: Named after the angulate tortoise or "rooipens" (Chersina angulata), a tortoise species found in dry areas and scrub forest in South Africa. The angulate tortoise is used as a food source by people in rural areas, and is also often kept as a pet.

Diagnosis: Similar to Neocamarosporium chichastianum [conidia (11-)15-19(-22) × (6-)8-9(-11) $\mu \mathrm{m}$ ], but conidia smaller.

Type: South Africa: Western Cape Province: Robben Island, on dead angulate tortoise shell, 25 May 2015, P.W. Crous (CBS H-23070 - holotype; CPC 27298 = CBS 142620 culture ex-type).

Description: Conidiomata solitary, pycnidial, immersed to erumpent, golden brown, 200-300 $\mu \mathrm{m}$ diam, with central ostiole, 25-35 $\mu \mathrm{m}$ diam; wall of 2-3 layers of brown textura angularis. Conidiophores reduced to conidiogenous cells, hyaline, smooth, subcylindrical, 3-6 × 2-4 $\mu \mathrm{m}$; proliferating several times percurrently at apex (conidiogenous cells dissolve at maturity). Conidia solitary, golden brown, thickwalled, smooth, broadly ellipsoidal to subcylindrical or irregular, transverse 3-septate, with 0-3 oblique septa, (10-) $12-13(-15) \times(5-) 6(-7) \mu \mathrm{m}$.

Culture characteristics: Colonies covering the dish in 2 wk, with fluffy aerial mycelium. On OA, MEA and PDA surface olivaceous grey to pale olivaceous grey, reverse olivaceous grey.

Notes: Neocamarosporium chersinae is introduced as a new species of Neocamarosporium (Crous et al. 2014b). Neocamarosporium is camarosporium-like in morphology, and can only be safely distinguished from this genus based on DNA data.

\section{Libertasomycetaceae Crous, fam. nov. MycoBank MB820911}

Etymology: Named after the genus Libertasomyces.
Classification: Libertasomycetaceae, Pleosporales, Dothideomycetes.

Diagnosis: Similar to Coniothyriaceae, but distinct in that ascomata are immersed in a brown stroma, and conidiomata are stromatic in culture.

\section{Type genus: Libertasomyces Crous \& Roets 2016.}

Description: Ascomata immersed in a brown stroma, becoming erumpent, breaking through the host surface, aggregated in clusters, with a central ostiole; wall of 6-10 layers of brown textura angularis. Pseudoparaphyses hyphal-like, intermingled among asci, hyaline, smooth, septate, anastomosing. Asci fasciculate, stipitate, hyaline, smooth, subcylindrical, bitunicate with ocular chamber, containing 8 ascospores. Ascospores fusoid-ellipsoidal, brown, verruculose with obtuse ends, muriformly septate, encased in a mucoid sheath. Conidiomata unilocular, stromatic, separate, globose, immersed, brown, opening via a central ostiole, exuding a brown conidial mass; wall of 3-6 layers of brown textura angularis. Conidiophores reduced to conidiogenous cells. Conidiogenous cells lining the inner cavity, hyaline, smooth, ampulliform to doliiform, with prominent periclinal thickening at the apex, or with tightly aggregated percurrent proliferations at the apex. Conidia solitary, golden brown, subcylindrical to ellipsoidal, straight to curved, 0-1-septate, constricted at median septum, apex obtuse, base truncate, with marginal frill, and longitudinal striations, or hyaline, smooth, granular, thin-walled, ellipsoidal, apex obtuse, base truncate to bluntly rounded, aseptata.

Genera included: Libertasomyces and Neoplatysporoides.

\section{Libertasomyces quercus Crous, sp. nov. MycoBank MB820912}

(Fig. 11)

Etymology: Named after the genus from which it was isolated, Quercus.

Diagnosis: Similar to Camarosporium quaternatum (conidia 18-34 × 9-16 $\mu \mathrm{m})$, but distinct in having smaller, uniformly brown conidia $(15-21 \times 6-10 \mu \mathrm{m})$. 

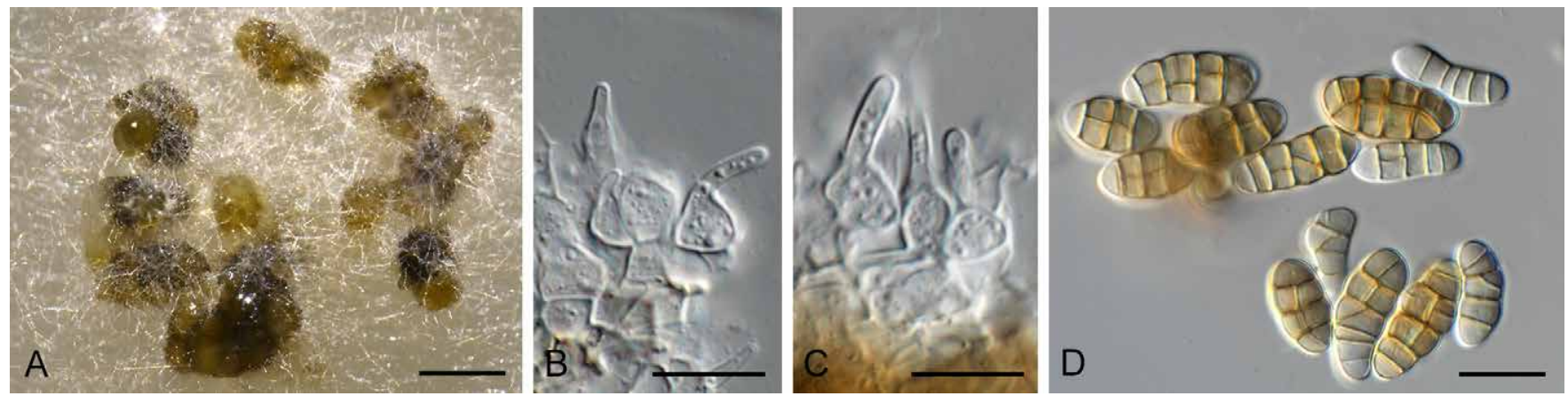

Fig. 11. Libertasomyces quercus (CBS 134.97). A. Conidiomata on OA. B, C. Conidiogenous cells. D. Conidia. Bars: $A=200 \mu m$, all others $=$ $10 \mu \mathrm{m}$.

Type: Spain: location unknown, on leaf litter of Quercus ilex (Fagaceae), 20 Jul. 1996, R.F. Castañeda (CBS H-23065 holotype; CBS 134.97 = INIFAT C96/108 - culture ex-type).

Description: Conidiomata brown, solitary or aggregated in clusters, stromatic, obovoid, 120-180 $\mu \mathrm{m}$ diam, with paler brown papillate ostiole, outer region covered with brown hyphae; wall of 6-12 layers of brown textura angularis. Conidiophores reduced to conidiogenous cells lining the inner cavity, hyaline, smooth, doliiform to ampulliform, 5-7 $\times 4-6 \mu \mathrm{m}$; proliferating percurrently at apex. Conidia solitary, brown, smooth, subcylindrical to broadly ellipsoidal, apex obtuse, base truncate to bluntly rounded, transversely 3-7-septate, becoming muriformly septate with vertical and oblique septa, (15-)17-19(-21) × (6-)7-8(-10) $\mu \mathrm{m}$.

Culture characteristics: Colonies flat, spreading, reaching 40 $\mathrm{mm}$ diam after $2 \mathrm{wk}$ with sparse to moderate aerial mycelium and even, lobate margins. On PDA surface umber with patches of salmon, reverse hazel. On OA surface dirty white with patches of umber.

Notes: Libertasomyces quercus (conidia 15-21 × 6-10 $\mu \mathrm{m}$ ) was originally identified as Camarosporium quaternatum (conidia 18-34 × 9-16 $\mu \mathrm{m}$, see above), but differs in conidial dimensions. Phylogenetically, it is better accommodated in Libertasomyces (based on L. myopori; Crous et al. 2016), which is pleomorphic, having a sexual morph, a camarosporium-like and a phoma-like synasexual morph.

Leptosphaeriaceae M.E. Barr, Mycotaxon 29: 503 (1987).

Type genus: Leptosphaeria Ces. \& De Not.1863.

\section{Querciphoma Crous, gen. nov.} MycoBank MB820913

Etymology: Named after the host on which it occurs, Quercus, and its phoma-like morphology.

Diagnosis: Morphologically similar to Phoma, but distinct in that conidiomata are eustromatic, uni- to multi-locular, and conidia become brown and verruculose with age.
Type species: Querciphoma carteri (Gruyter \& Boerema) Crous 2017.

Description: Conidiomata pycnidial, globose, eustromatic, uni- to multi-locular, with 1-3 papillate ostioles, medium brown, outer surface covered in short, brown, verruculose, septate setae with obtuse ends; wall of 3-6 layers of brown textura angularis. Conidiophores reduced to conidiogenous cells lining inner cavity, hyaline, smooth, doliiform; phialidic with periclinal thickening at the apex. Conidia solitary, hyaline, smooth, guttulate, broadly ellipsoidal, aseptate, becoming brown, verruculose with age.

\section{Querciphoma carteri (Gruyter \& Boerema) Crous, comb. nov.}

MycoBank MB820914

(Fig. 12)

Basionym: Phoma carteri Gruyter \& Boerema, Persoonia 17: 547 (2002) [“2001”].

Synonyms: Coniothyrium carteri (Gruyter \& Boerema) Verkley \& Gruyter, Stud. Mycol. 75: 23 (2013) [“2012”].

Pyrenochaeta minuta J.C. Carter, Bull. Illinois Nat. Hist. Surv. 21: 214 (1941).

Description: Conidiomata pycnidial, globose, eustromatic, uni- to multi-locular, with 1-3 papillate ostioles, 150-250 $\mu \mathrm{m}$ diam, medium brown, outer surface covered in short, brown, verruculose, septate setae with obtuse ends, to $80 \mu \mathrm{m}$ tall; wall of 3-6 layers of brown textura angularis. Conidiophores reduced to conidiogenous cells lining inner cavity, hyaline, smooth, doliiform, 4-6 × 4-5 $\mu \mathrm{m}$; phialidic with periclinal thickening at apex. Conidia solitary, hyaline, smooth, guttulate, broadly ellipsoidal, aseptate, becoming brown, verruculose with age, apex obtuse, base truncate to bluntly rounded, (3.5-)4-5(-6) × 3(-3.5) $\mu \mathrm{m}$.

Culture characteristics: Colonies flat, spreading, reaching 50 $\mathrm{mm}$ diam after $2 \mathrm{wk}$, with sparse to moderate aerial mycelium, and smooth, lobate margins. On PDA surface umber, reverse chestnut. On OA surface umber.

Material examined: Germany: former West-Germany: on leaves and twigs of Quercus robur (Fagaceae), H. Schill (CBS H-23071, culture CBS 105.91). - The Netherlands: PD Lisse, on Quercus sp., date and collector unknown (PD 84/74 = CBS 101633). 

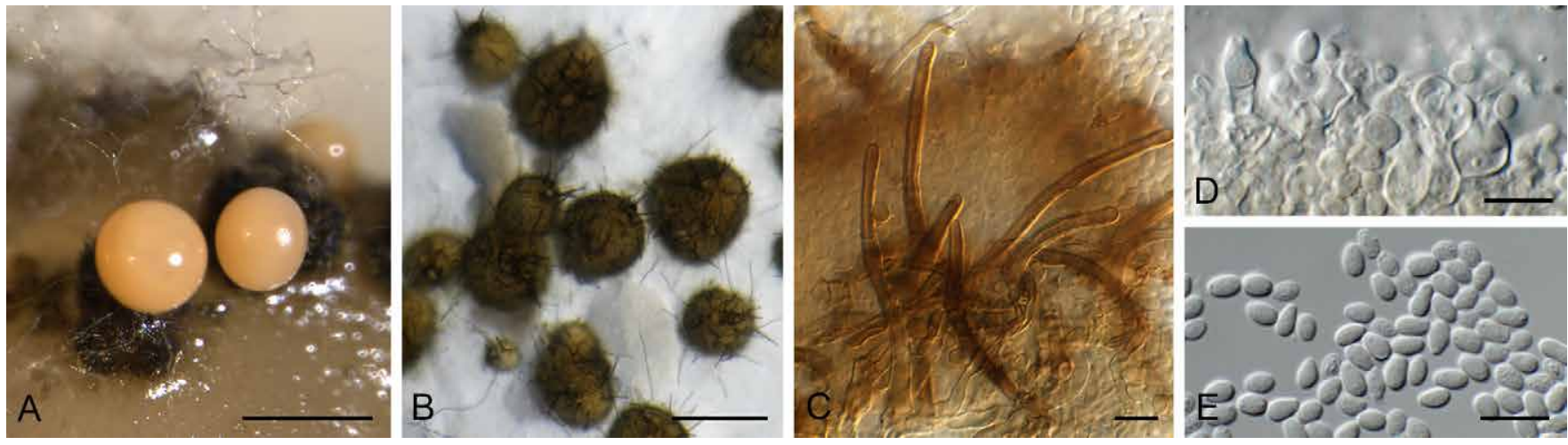

Fig. 12. Querciphoma carteri (CBS 105.91). A. Conidiomata on PDA. B. Conidiomata on SNA. C. Setae. D. Conidiogenous cells. E. Conidia. Bars: $A, B=250 \mu \mathrm{m}$, all others $=10 \mu \mathrm{m}$.

Notes: This isolate was deposited as Phoma sp., and reclassified as Coniothyrium carteri (based on Phoma carteri; see Boerema et al. 2004) by De Gruyter et al. (2013). However, as shown here, it clusters separately from Coniothyrium s.str. (having brown, 1-septate conidia), and is best placed in a separate genus.

Didymosphaeriaceae Munk, Dansk bot. Ark. 15: 128 (1953).

Type genus: Didymosphaeria Fuckel 1870.

\section{Paracamarosporium mamanes (Crous) Crous, comb.} nov.

MycoBank MB820916

Basionym: Camarosporium mamanes Crous, Fungal Planet No. $5: 1$ (2006).

Type: USA: Hawaii: Saddle Road, on stems of Sophora chrysophylla (Leguminosae), Aug. 2005, W. Gams \& Y. Degawa (CBS H-19775 - holotype; CPC 12252 = CBS 120031, CPC 12253-12254 - cultures ex-type).

Notes: Camarosporium mamanes is allocated to the genus Paracamarosporium based on its phylogenetic position. However, the distinction between Paracamarosporium and Pseudocamarosporium is highly debatable (Wijayawardene et al. 2014), and although we could separate them here based on ITS sequence data, the genera cannot be separated based on LSU data, and appear to represent a single genus, Paracamarosporium. Furthermore, there have also been an excessive number of species introduced in the genus, which are not supported based on their DNA barcodes.

\section{DOTHIORA COMPLEX}

Dothideaceae Chevall., Fl. gén. env. Paris 1: 446 (1826); as "Dothideae".

Type genus: Dothidea Fr. 1818.
Dothiora Fr., Summa veg. Scand. 2: 418 (1849).

Synonyms: Dothichiza Lib. ex Roum., Fungi Selecti Gallici Exs. cent. 7, no. 627 (1880).

Coleonaema Höhn., Mitt. Bot. Lab. TH Wien 1(3): 95 (1924).

Cylindroseptoria Quaedvl., et al., Stud. Mycol. 75: 358 (2013).

Additional synonyms: See Crous \& Groenewald (2016).

Classification: Dothideaceae, Dothideales, Dothideomycetes.

Current generic circumscription: Ascostromata immersed to erumpent, pulvinate to globose, black, multi-loculate; wall of dark brown textura angularis. Locules globose to subglobose, broadly rounded or papillate with central ostiole. Pseudoparaphyses absent. Asci 8- or more spored, bitunicate, fissitunicate, oblong to clavate, pedicellate, with a small ocular chamber. Ascospores biseriate to multi-seriate, septate, constricted at the primary median septum, at times with a vertical septum, hyaline, rarely pale brown, obovate to ellipsoidal to fusoid, often inequilateral or slightly curved, smooth, at times with a thin mucoid sheath. Conidiomata pycnidial, separate, or aggregated in a stroma. Conidiophores reduced to conidiogenous cells lining the inner cavity, hyaline, smooth, ampulliform to doliiform, phialidic. Conidia aseptate, hyaline, smooth, subcylindrical to ovoid or oblong. Hyphae becoming brown, verruculose and constricted at septa, giving rise to a hormonema-like synasexual morph.

Type species: Dothiora pyrenophora (Fr.) Fr. 1849.

\section{Dothiora cactacearum Crous, sp. nov.} MycoBank MB820917

(Fig. 13)

Etymology: Named after the host family from which the fungus was isolated, Cactaceae.

Diagnosis: Morphologically distinct from other species of Dothiora in that conidia become brown and roughened with age.

Type: USA: Texas: Austin, on phyllodes of Cactaceae, 2013, P.W. Crous (CBS H-23074 - holotype; CPC 15585 = CBS 142492 - culture ex-type, CPC 15587). 

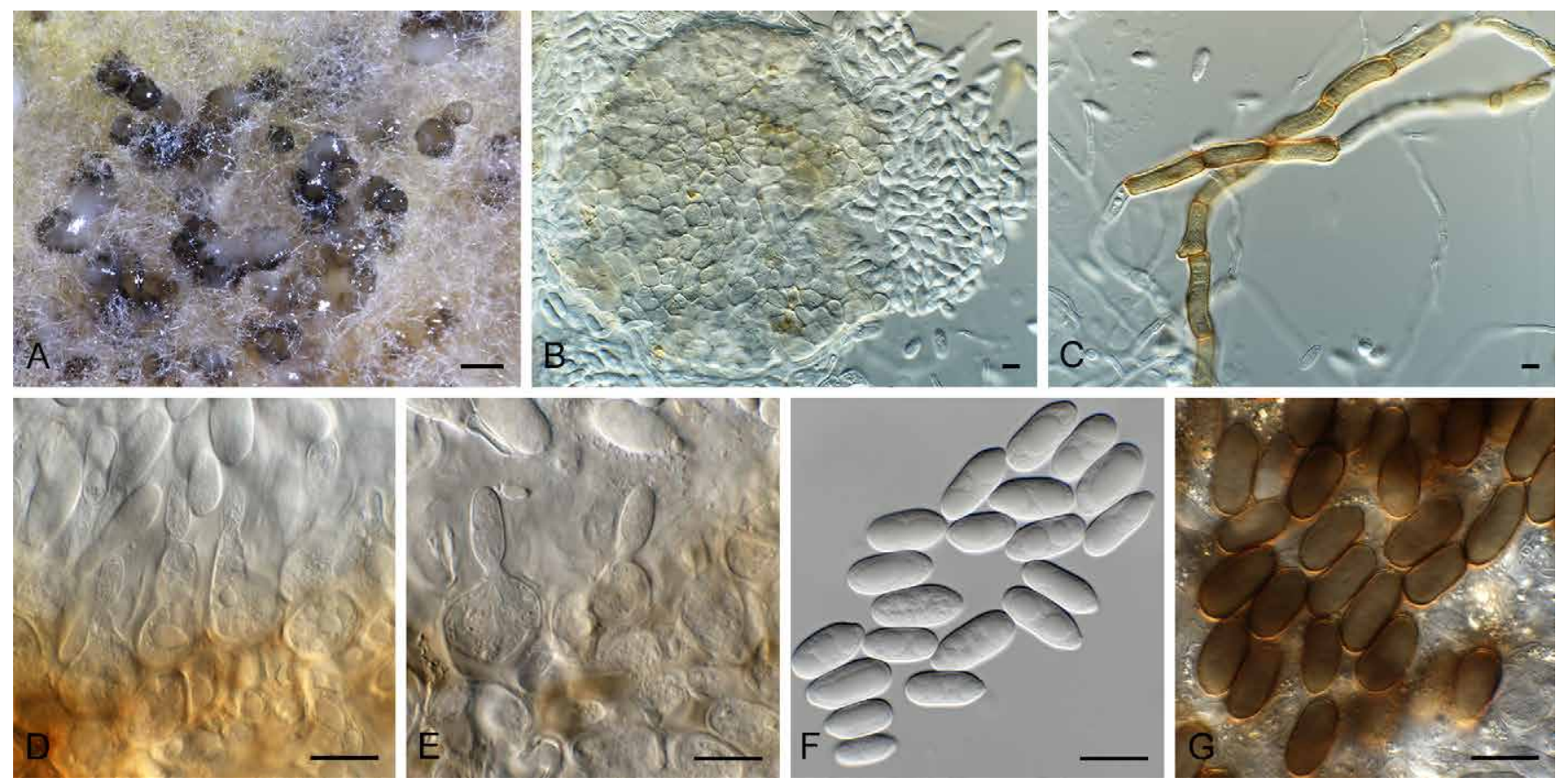

Fig. 13. Dothiora cactacearum (CPC 15585). A. Conidiomata on OA. B. Conidioma. C. Brown hyphae forming on SNA. D, E. Conidiogenous cells. F. Conidia. G. Conidia turning brown and verruculose with age. Bars: $A=150 \mu \mathrm{m}$, all others $=10 \mu \mathrm{m}$.

Description: Conidiomata separate, erumpent, pycnidial, globose, medium brown, 150-300 $\mu \mathrm{m}$ diam, with a central ostiole, exuding a creamy conidial mass; wall of 3-6 layers of brown textura angularis. Conidiophores reduced to conidiogenous cells lining the inner cavity, hyaline, smooth, ampulliform to doliiform, 7-15 × 7-15 $\mu \mathrm{m}$, phialidic, at times with percurrent proliferation and prominent collarette. Conidia hyaline, smooth, guttulate, subcylindrical to broadly ellipsoidal, apex obtuse, tapering to a truncate, protruding hilum, 2-3 $\mu \mathrm{m}$ diam, (12-)14-17(-19) × (5-)6-7.5(-8) $\mu \mathrm{m}$ (av. $15 \times 7 \mu \mathrm{m})$; conidia becoming brown and roughened with age. Hyphae becoming brown, verruculose and constricted at the septa.

Culture characteristics: Colonies flat, spreading, with sparse to moderate aerial mycelium, and even, lobate margin, reaching $40 \mathrm{~mm}$ diam after $2 \mathrm{wk}$ at $25^{\circ} \mathrm{C}$. On MEA surface umber, reverse luteous; on PDA surface fawn, reverse isabelline; on OA surface isabelline.

\section{Dothiora pyrenophora (Fr.) Fr., Summa veg. Scand.} 2: 418 (1849).

(Fig. 14)

Basionym: Dothidea pyrenophora Fr., Kongl. Vetensk. Acad. Handl. 40: 88 (1819) : Fr., Syst. mycol. 2(2): 552 (1823).

Type: Sweden: without locality, on dead branches of Sorbus sp. (Rosaceae), E.M. Fries (no original material found). Germany: on twig of Sorbus aucuparia, 27 Apr. 2016, R.K. Schumacher (CBS H-23073 - neotype designated here, MBT376258; CPC 30634, CPC 30632 = CBS 142621cultures ex-neotype).

Description: Ascostromata solitary to aggregated, black, immersed to erumpent, unilocular, to $400 \mu \mathrm{m}$ diam, elliptical, pulvinate, opening by an irregular pore, upper layer dissolving with age; wall of 6-10 layers of brown textura angularis. Asci bitunicate, hyaline, oblong to subcylindrical, short stipitate, 8-spored, with apical apiculus, 2-3 $\mu \mathrm{m}$ diam, 90-120 × 14-17 $\mu \mathrm{m}$. Ascospores bi- to triseriate in ascus, hyaline, smooth, at times turning yellow-brown with age, fusiform, inequilateral, slightly curved, with prominent mucoid sheath when young (in water), dissolving at maturity, (5-)5(-8) transversely septate, prominently constricted at primary septum, with oblique or vertical septa in central cells, (22-)25-30(-35) × (7-)8 $\mu \mathrm{m}$; ascospores directly giving rise to asexual morph via budding, with ascomata transforming with age into large conidiomata, with apical opening completely dissolving. Conidiomata immersed to erumpent, pycnidial, black, globose, to $300 \mu \mathrm{m}$ diam, separate or gregarious, unilocular; wall of 3-6 layers of brown textura angularis. Conidiophores lining the inner cavity, reduced to conidiogenous cells. Conidiogenous cells hyaline, smooth, doliiform to ampulliform, 4-9 × 4-6 $\mu \mathrm{m}$, with minute periclinal thickening at apex. Conidia solitary, aseptate, hyaline, smooth, ovate to ellipsoidal, with minute guttules, subobtuse apex, and truncate hilum, (5-)7-8(-9) × (3-)4 $\mu \mathrm{m}$.

Culture characteristics: Colonies flat, spreading, with sparse aerial mycelium and feathery, undulate margins. On PDA, MEA and OA surface and reverse iron-grey.

Other material examined: Germany: on twig of Sorbus aucuparia, 27 Apr. 2016, R.K. Schumacher (CBS H-23072).

Notes: The taxonomic position of the several species occurring on Sorbus require further clarification based on fresh material. One culture identified as Dothiora sorbi (CBS 742.71) was included in this study, and clustered slightly apart from $D$. pyrenophora (nine bp different on ITS), suggesting 

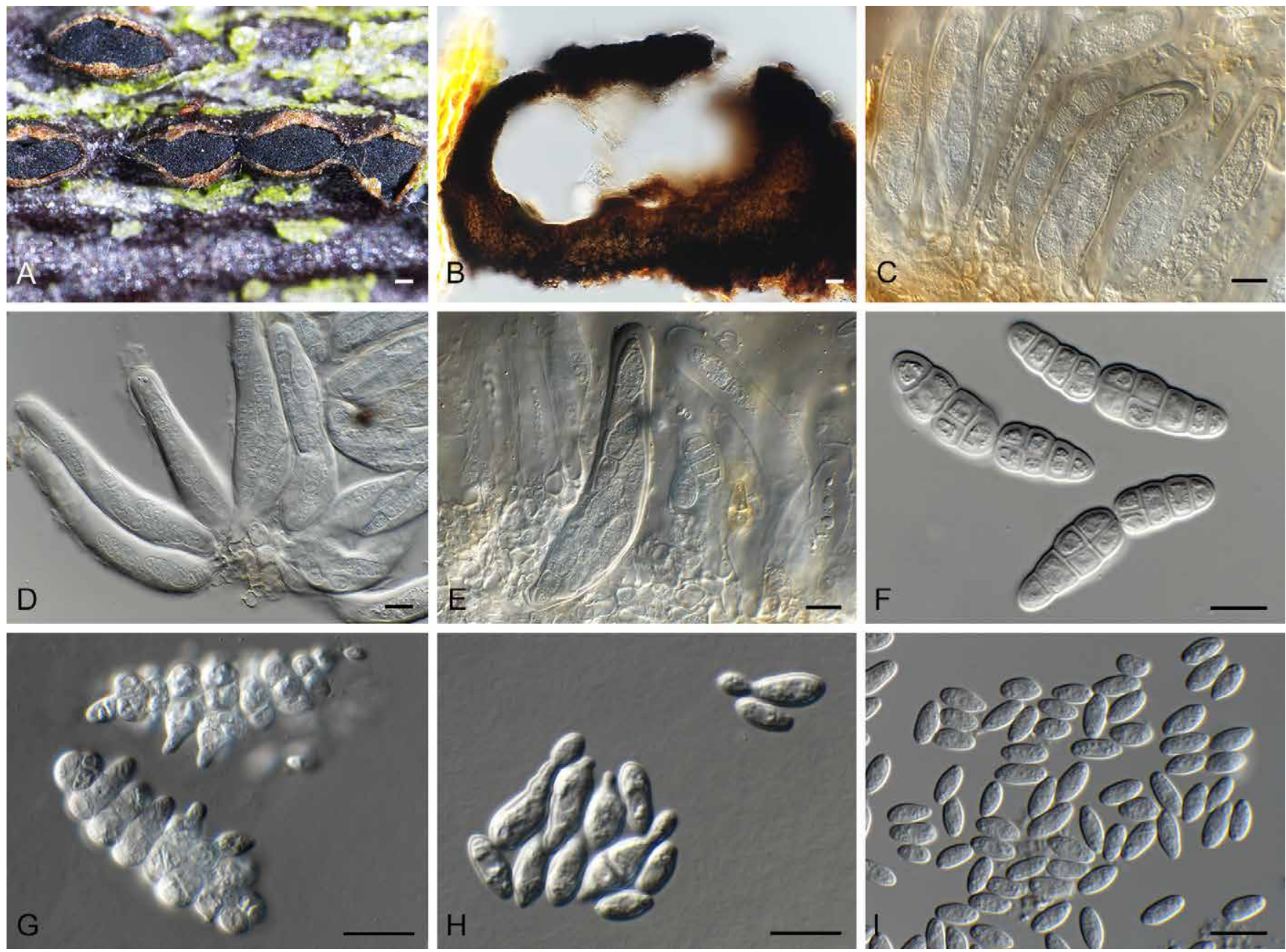

Fig. 14. Dothiora pyrenophora (CPC 30632). A. Ascostroma on twig. B. Section through ascoma. C-E. Asci. F. Ascospores. G. Ascospores undergoing microcyclic conidiation. H. Conidia undergoing budding. I. Conidia. Bars: $A, B=200 \mu \mathrm{m}$, all others $=10 \mu \mathrm{m}$.

that these may very well be two closely related, but distinct species.

Genera such as Sydowia Bres.1895 and Pringsheimia Schulzer 1866 appear to represent younger generic names within Dothideaceae, based on their morphological similarity, and the formation of Dothichiza and hormonemalike morphs in culture, which are commonly observed in the family (Froidevaux 1972, Sivanesan 1984, Crous \& Groenewald 2016). Another confusing aspect of many of the taxa in this family is that young ascospores can be hyaline and uniseptate, but become brown and muriformly septate with age (e.g. Dothidea ribesia, Fig. 15), which complicate the generic delimitation within the family (Thambugala et al. 2014), and identification keys based on immature material.

Species of Dothiora are commonly isolated from dead branches of woody hosts (Sivanesan 1984), while Crous \& Groenewald (2016) also reported some species from dead leaves and fruit of diverse hosts, suggesting that it is a saprobe, possibly acting as a weak pathogen on stressed plant tissues.

\section{ACKNOWLEDGEMENTS}

We acknowledge Laszlo Bartalos and René Jarling for sending us specimens of Camarosporium and similar taxa examined in the present study. We are also thankful to René K. Schumacher for making many valuable collections available to be included in this study, and for bringing the problem related to the nomenclature of Camarosporium to our attention.

\section{REFERENCES}

Boerema GH, De Gruyter J, Noordeloos ME, Hamers MEC (2004) Phoma Identification Manual: Differentiation of specific and infraspecific taxa in culture. Wallingford: CABI Publishing.

Carbone I, Kohn LM (1999) A method for designing primer sets for speciation studies in filamentous ascomycetes. Mycologia 91: 553-556.

Cheewangkoon R, Crous PW, Hyde KD, Groenewald JZ, To-anan C (2008) Species of Mycosphaerella and related anamorphs on Eucalyptus leaves from Thailand. Persoonia 21: 77-91.

Crous PW, Gams W, Stalpers JA, Robert V, Stegehuis G (2004) MycoBank: an online initiative to launch mycology into the 21st century. Studies in Mycology 50: 19-22.

Crous PW, Giraldo A, Hawksworth DL, Robert V, Kirk PM, et al. (2014a) The Genera of Fungi: fixing the application of type species of generic names. IMA Fungus 5: 141-160.

Crous PW, Groenewald JZ (2016) They seldom occur alone. Fungal Biology 120: 1392-1415. 

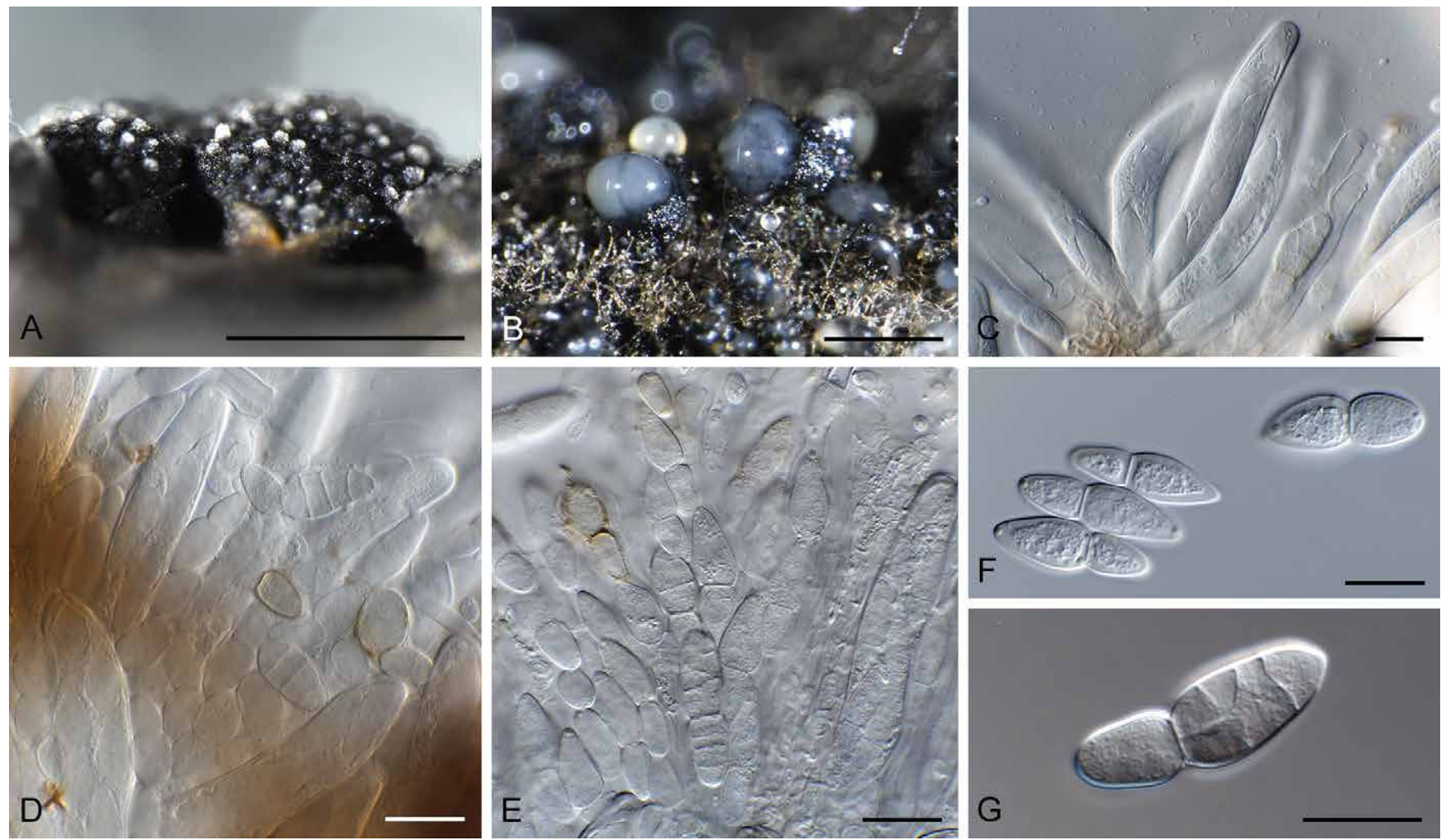

Fig. 15. Dothidea ribesia (CPC 30638). A. Ascostromata on twig. B. Ostioles with exuding ascospores. C-E. Asci with ascospores that become muriformly septate. F. One-septate ascospores. G. Muriformly septate ascospore. Bars: $A=3 \mathrm{~mm}, B=100 \mu \mathrm{m}$, all others $=10 \mu \mathrm{m}$.

Crous PW, Hawksworth DL, Wingfield MJ (2015a) Identifying and naming plant-pathogenic fungi: past, present, and future. Annual Review of Phytopathology 53: 246-267.

Crous PW, Shivas RG, Quaedvlieg W, van der Bank M, Zhang Y, et al. (2014b) Fungal Planet Description Sheets: 214-280. Persoonia 32: 184-306.

Crous PW, Slippers B, Wingfield MJ, Rheeder J, Marasas WFO, et al. (2006) Phylogenetic lineages in the Botryosphaeriaceae. Studies in Mycology 55: 235-253.

Crous PW, Verkley GJM, Groenewald JZ, Samson RA (eds) (2009) Fungal Biodiversity. [CBS Laboratory Manual Series no.1.] Utrecht: CBS-KNAW Fungal Biodiversity Centre.

Crous PW, Wingfield MJ, Guarro J, Cheewangkoon R, van der Bank $\mathrm{M}$, et al. (2013). Fungal Planet description sheets: 154-213. Persoonia 31: 188-296.

Crous PW, Wingfield MJ, Guarro J, Hernández-Restrepo M, Sutton DA, et al. (2015b) Fungal Planet description sheets: 320-370. Persoonia 34: 167-266.

Crous PW, Wingfield MJ, Park RF (1991) Mycosphaerella nubilosa a synonym of M. molleriana. Mycological Research 95: 628-632.

Crous PW, Wingfield MJ, Richardson DM, Leroux JJ, Strasberg D, et al. (2016). Fungal Planet description sheets: 400-468. Persoonia 36: 316-458.

De Gruyter J, Woudenberg JHC, Aveskamp MM, Verkley GJM, Groenewald JZ, Crous PW (2013) Redisposition of Phoma-like anamorphs in Pleosporales. Studies in Mycology 75: 1-36.

De Hoog GS, Gerrits van den Ende AHG (1998) Molecular diagnostics of clinical strains of filamentous basidiomycetes. Mycoses 41: 183-189.

Froidevaux L (1972) Contribution à l'étude des Dothioracées (Ascomycètes). Nova Hedwigia 23: 679-734.
Glass NL, Donaldson G (1995). Development of primer sets designed for use with PCR to amplify conserved genes from filamentous ascomycetes. Applied and Environmental Microbiology 61: 1323-1330.

Hawksworth DL, Crous PW, Redhead SA, Reynolds DR, Samson RA et al. (2011) The Amsterdam Declaration on Fungal Nomenclature. IMA Fungus 2: 105-112.

Hawksworth DL, Halici MG, Kocakaya Z, Kocakaya M (2016) Henfellra muriformis gen. et sp. nov., a new dictyosporous pycnidial fungus on Candelariella, with a key to the lichenicolous fungi known from that genus. Herzogia 29: 329-336.

Hazslinszky F (1865) Beitrag zur Kenntnis der Sphärien des Lyciums. Verhandlungen der Kaiserlich-Königlichen ZoologischBotanischen Gesellschaft in Wien 15: 447-452.

Kirk PM, Stalpers JA, Braun U, Crous PW, Hansen K, et al. (2013) A without-prejudice list of generic names of fungi for protection under the International Code of Nomenclature for algae, fungi and plants. IMA Fungus 4: 381-443.

O'Donnell K, Cigelnik E (1997) Two divergent intragenomic rDNA ITS2 types within a monophyletic lineage of the fungus Fusarium are nonorthologous. Molecular Phylogenetics and Evolution 7: 103-116.

O’Donnell K, Kistler HC, Cigelnik E, Ploetz RC (1998) Multiple evolutionary origins of the fungus causing Panama disease of banana: concordant evidence from nuclear and mitochondrial gene genealogies. Proceedings of the National Academy of Sciences, USA 95: 2044-2049.

Quaedvlieg W, Groenewald JZ, de Jesús Yáñez-Morales M, Crous PW (2012) DNA barcoding of Mycosphaerella species of quarantine importance to Europe. Persoonia 29: 101-115.

Robert V, Vu D, Amor ABH, van de Wiele N, Brouwer C, et al. (2013) MycoBank gearing up for new horizons. IMA Fungus 4: 371-379. 
Rehner SA, Buckley E (2005) A Beauveria phylogeny inferred from nuclear ITS and EF1- $\alpha$ sequences: evidence for cryptic diversification and links to Cordyceps teleomorphs. Mycologia 97: 84-98.

Rayner RW (1970) A Mycological Colour Chart. Kew: Commonwealth Mycological Institute.

Rossman AY, Adams GC, Cannon PF, Castlebury LA, Crous PW, et al. (2015) Recommendations of generic names in Diaporthales competing for protection or use. IMA Fungus 6: 145-154.

Saccardo PA (1883) Sylloge Pyrenomycetum. Sylloge Fungorum 2: 1-813.

Sivanesan A (1984) The Bitunicate Ascomycetes and their Anamorphs. Vaduz: J. Cramer.

Smith H, Wingfield MJ, Crous PW, Coutinho TA (1996) Sphaeropsis sapinea and Botryosphaeria dothidea endophytic in Pinus spp. and Eucalyptus spp. in South Africa. South African Journal of Botany 62: 86-88.

Sutton BC (1980) The Coelomycetes: fungi imperfecti with pycnidia, acervuli, and stromata. Kew: Commonwealth Mycological Institute.

Swofford DL (2003) PAUP*: phylogenetic analysis using parsimony (*and other methods). Version 4. Sunderland, MA: Sinauer Associates.
Thambugala KM, Ariyawansa HA, Li Y-M, Boonmee S, Hongsanan S, et al. (2014) Dothideales. Fungal Diversity 68: 105-158.

Vilgalys R, Hester M (1990) Rapid genetic identification and mapping of enzymatically amplified ribosomal DNA from several Cryptococcus species. Journal of Bacteriology 172: 4238-4246.

White TJ, Bruns T, Taylor J (1990) Amplification and direct sequencing of fungal ribosomal RNA genes for phylogenetics. In: PCR Protocols: a guide to molecular methods and applications (Innis MA, Gelfand DH, Sninsky JJ, White JW, eds): 315-322. San Diego: Academic Press.

Wijayawardene NN, Hyde KD, Bhat DJ, Camporesi E, Schumacher RK, et al. (2014) Camarosporium-like species are polyphyletic in Pleosporales; introducing Paracamarosporium and Pseudocamarosporium gen. nov. in Montagnulaceae. Cryptogamie, Mycologie 35: 177-198.

Wijayawardene NN, Hyde KD, Wanasinghe DN, Papizadeh M, Goonasekara ID, et al. (2016) Taxonomy and phylogeny of dematiaceous coelomycetes. Fungal Diversity 77: 1-316. 